\title{
Oblique elastic plate impact on thin liquid layer
}

Cite as: Phys. Fluids 32, 062101 (2020); https://doi.org/10.1063/5.0007121

Submitted: 11 March 2020 . Accepted: 12 May 2020 . Published Online: 01 June 2020

T. I. Khabakhpasheva (D), and A. A. Korobkin (D)

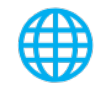

\section{ARTICLES YOU MAY BE INTERESTED IN}

Fluvial instabilities

Physics of Fluids 32, 061301 (2020); https://doi.org/10.1063/5.0010038

The singular hydrodynamic interactions between two spheres in Stokes flow

Physics of Fluids 32, 062001 (2020); https://doi.org/10.1063/5.0009053

Droplet deformation and breakup in shear flow of air

Physics of Fluids 32, 052109 (2020); https://doi.org/10.1063/5.0006236 


\title{
Oblique elastic plate impact on thin liquid layer
}

Cite as: Phys. Fluids 32, 062101 (2020); doi: 10.1063/5.0007121

Submitted: 11 March 2020 - Accepted: 12 May 2020 •

Published Online: 1 June 2020

\section{T. I. Khabakhpasheva ${ }^{1, a)}$ (iD) and A. A. Korobkin ${ }^{1,2, b)}$ (iD}

\author{
AFFILIATIONS \\ ${ }^{1}$ Lavrentyev Institute of Hydrodynamics, pr. Lavrentyeva 15, Novosibirsk 630090, Russia \\ ${ }^{2}$ University of East Anglia, Norwich NR4 7TJ, United Kingdom
}

a) Author to whom correspondence should be addressed: tana@hydro.nsc.ru

${ }^{b)}$ Electronic mail: a.korobkin@uea.ac.uk

\begin{abstract}
The present study is concerned with possible mechanisms of air entrainment in a thin liquid layer caused by oblique impact of a deformable body on the layer. The two-dimensional unsteady problem of oblique elastic plate impact is considered within the thin-layer approximation for the first time. The plate deflection is described by the Euler beam equation. The plate edges are free of stresses and shear forces. The plate deflections are comparable with the liquid layer thickness. It is revealed in this paper that, for a stiff plate, the initial impact by the trailing edge makes the plate rotate with the leading plate edge entering water before the wetted part of the plate arrives at this edge. The air cavity trapped in such cases can be as long as $40 \%$ of the plate length. For a flexible plate, the impact does not cause the plate rotation. However, the dry part of the plate in front of the advancing wetted region is deflected toward the liquid layer also trapping the air. The numerical results are presented for elastic and rigid motions of the plate, hydrodynamic pressure in the wetted part of the plate, position of this wetted part, and the flow beneath the plate.
\end{abstract}

Published under license by AIP Publishing. https://doi.org/10.1063/5.0007121

\section{INTRODUCTION}

The unsteady two-dimensional and nonlinear problem of oblique impact by an elastic plate onto a thin liquid layer is considered. The edges of the plate are free of bending stresses and shear forces [see Fig. 1(a)]. This study is motivated by the experiments on droplet deposition in annular gas-liquid flow and mass exchange between the gas core and the liquid film (Cherdantsev et al., 2017). Bubbles entrapped in the liquid film were observed for some conditions of oblique droplet impacts onto the film. The bubbles were created during the impacts. Then, some of the bubbles collapsed behind the impacting droplet, but others survived inside the liquid film. Both the number of bubbles entrapped in the film and their total volume were found to be dependent on the diameter of the impacting droplet. Typical conditions of droplet impacts, which led to bubble entrainment in the thin liquid layer, were as follows: the diameter of the droplet was of order of $1 \mathrm{~mm}$, the liquid layer thickness was of order of $0.2 \mathrm{~mm}$, the horizontal speed of the droplets was of order of $30 \mathrm{~m} / \mathrm{s}$, and the vertical speed was of order of $1 \mathrm{~m} / \mathrm{s}$. Bubble entrainment was observed, for example, for a droplet of diameter $0.71 \mathrm{~mm}$ impacting a thin film at a horizontal speed of $20 \mathrm{~m} / \mathrm{s}$. The rate of bubble entrainment was high and could not be explained by the air-cushion effect, which is less significant for oblique liquid impacts [see Hicks and Purvis (2010; 2011)].

The present study aims at identifying some possible mechanisms of bubble entrainment in a thin liquid layer as a result of oblique impact onto the layer by a deformable body, which mimics a liquid droplet. The simplest configuration with free-free elastic thin plate is considered. One may assume that the air can be trapped (a) in front of the impacting body due to the body deformation toward the liquid and jetting, (b) under the body because of the body vibration and possible cavitation caused by the impact, and (c) behind the body because of complex and oscillatory behavior of the wake. These potential mechanisms of air entrainment could be important also at larger scale of high-speed boats and ditching aircraft.

We assume that (a) the penetration of the body into the liquid layer is comparable with the thickness of the layer and (b) the horizontal dimension of the problem, which is the projection of the plate onto the layer, is much greater than the layer thickness. These assumptions make it possible to describe the flow between the impacting body and the bottom of the liquid layer within the thin-layer approximation [see Korobkin (1995; 1999)] using certain matching conditions at the edges of the wetted part of the body surface. In the two-dimensional problem of oblique plate impact, the 

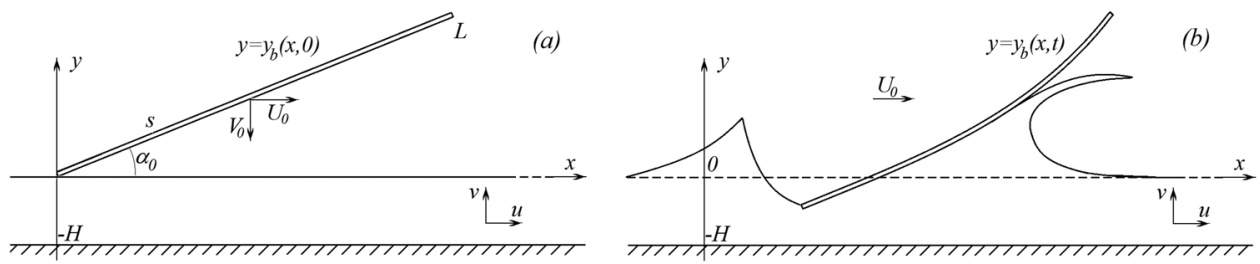

FIG. 1. Oblique impact by elastic plate (a) Initial position of the plate and notation. (b) Scheme of the flow and plate deflection during the impact. The position of the plate is described by the equation $y=y_{b}(x, t)$.

matching condition at the leading edge of the wetted part of the plate surface describes the jetting there [see Fig. 1(b)] and the matching condition at the trailing edge of the wetted surface describes the liquid separation from the plate surface. The liquid separation may occur either from the left edge of the plate [see Fig. 1(b)] or from the inner part of the smooth plate surface. In the latter case, the position of the separation point is determined using the BrillouinVillat condition (Birkhoff and Zarantonello, 1957; Khabakhpasheva and Korobkin, 2013a). The positions of both the leading and trailing edges of the wetted part of the plate depend on both the plate motions and elastic deflections.

The problem of oblique impact by an elastic plate with freefree edges was studied by Reinhard et al. (2013) for deep water using the Wagner theory of water impact with a wake behind the plate. The presence of the wake changes the pressure distribution along the wetted part of the plate, as well as the length and the position of this wetted part. In contrast to the deep water impact problem, the wake on shallow water behind the elastic plate does not affect in the leading order the hydrodynamic pressure under the plate because the horizontal speed of the plate and the speed of the generated flow are much higher than the critical speed of the wave propagation in the thin liquid layer. Reinhard (2013) studied both rigid and elastic plate impacts with different conditions of the flow separation at the trailing edge. Moradi et al. (2018) applied the analysis by Reinhard (2013) to other edge conditions. A linear hydrodynamic model of a rigid plate impact onto a thin liquid layer was studied by Liu (2017). Such a linear model is applicable for small penetrations of the plate into the liquid layer, the so-called skimming impact.

The shallow water model of water impact was developed by Korobkin $(1995 ; 1999)$ and generalized to elastic vertical impacts by Khabakhpasheva (2009; 2015) for two-dimensional cylindrical shells and spherical shells correspondingly and to oblique impacts and three-dimensional impacts by smooth rigid bodies by Batyaev and Khabakhpasheva (2013; 2016). Tkacheva (2008; 2013) investigated vertical impacts by a structure with an elastic flat bottom on a thin liquid layer without inclination of the bottom and with a small inclination angle.

Two-dimensional oblique impact by a rigid body with a smooth surface on a thin liquid layer was studied by Khabakhpasheva and Korobkin (2013a; 2013b) within the non-linear shallow water approximation. Calculations were performed for an elliptic cylinder with multiple impacts on the liquid layer. The penetration of the cylinder into the liquid layer was comparable with the thickness of the layer. The cylinder was "walking" on water with its rotation being as important as the vertical motion of the cylinder. The horizontal component of the hydrodynamic force acting on the body was shown to be negligible within the thin-layer approximation. The elliptic shape of a body (ellipsoid in 3D) is a preferable shape for multiple skipping from the water surface. Belden et al. (2016) and Hurd et al. (2019) discovered that soft spheres naturally take ellipsoidal shapes during their oblique impacts on deep water. Hurd et al. (2019) wrote "The behavior is characterized by the sphere moving nearly parallel to the water surface with the major axis tips dipping below the water surface with each rotation while the shorter sides pass just above, giving the impression that the sphere is walking across the water surface."

We are unaware of experiments with elastic plates or other elastic bodies impacting obliquely on a thin liquid layer. Experiments with three-dimensional rectangular plate impacting deep water at high horizontal speed were performed by Iafrati (2016). The plate was rigid enough and elastic deflections of the plate were not studied. The conditions of his experiments represented those of aircraft emergency landing on water. The experiments were concerned with three-dimensional effects and the motion of the pressure peak along the plate. The interpretation of the experimental results were supported by fully nonlinear two-dimensional solution within the potential flow model. More recently, Iafrati and Grizzi (2019) studied experimentally oblique impacts of double curvature panels on deep water with the horizontal speed of the panels ranging from $21 \mathrm{~m} / \mathrm{s}$ to $45 \mathrm{~m} / \mathrm{s}$. The authors wrote "Test data highlight different cavitation and/or ventilation modalities which are strongly dependent on the horizontal velocity, with substantial changes in the flow features occurring with velocity variations of few meters per second. For the specimen considered here, the inception of the cavitation is found at about $30 \mathrm{~m} / \mathrm{s}$, confirming that scaled model tests performed at small horizontal velocities are unable to capture the hydrodynamics correctly. By analyzing pressure data, underwater movies, and force measurements, it is shown that the transition from the cavitation to ventilation condition has a significant effect on the longitudinal distribution of the loading which, together with inertia, aerodynamic loads, and engine thrust, governs the aircraft dynamics." Elastic effects were not studied in these experiments as well. However, the curvature of the rigid panels can be considered as mimicking the complicated dynamics of elastic surfaces during their impacts on water, which defines conditions of cavitation under the panel.

Vertical impacts on deep and shallow waters have some similarities but they are also different in several important aspects. Relations between elastic body impacts on deep and shallow water were studied by Korobkin and Khabakhpasheva (2013). These authors investigated elastic responses of wedges and shells during water impact and concluded that "... the presence of the bottom increases stresses in an elastic structure entering the water layer. The increase in stresses caused by the bottom is significant only for very shallow layers." Dependencies of the impact pressures and conditions of cavitation on the depth of water were not studied. 
A nonlinear model of oblique elastic impact on a thin liquid layer is formulated and investigated in this paper for the first time. The governing equations of the plate both rigid and elastic motions and the liquid thin-layer flow beneath the plate are derived in Sec. II. It is shown in Sec. II C how to couple the structural and hydrodynamic equations when the elastic deflections of the plate are of the order of the thickness of the liquid layer. The problem is reduced to a system of non-linear ordinary differential equations in Sec. III. The system is written with respect to the principal coordinates of both rigid and elastic modes of the plate motions, the coordinate of the leading edge of the wetted part of the plate, and the flow velocity at this edge. The numerical algorithm of the problem is described in Sec. IV. The initial conditions are derived for the coupled problem where the contact region starts from a single point. It is explained how the Brillouin-Villat condition is imposed at the trailing edge of the contact region. A non-linear equation is derived for the coordinate of the trailing edge together with the derivative of this equation with respect to the unknown coordinate in order to apply Newton's method for solving this equation. The obtained numerical results are presented in Sec. V for the motions of the plate, distributions of the hydrodynamic pressure in the contact region, the positions of the edges of the contact region and their elevations above the liquid bottom, maximum strain and its position as functions of time, and the flow speeds at the edges. The horizontal speed of the flow, the initial inclination angle of the plate, and its rigidity were varied to investigate different scenarios of the impacts. The conclusions are drawn and future work is discussed in Sec. VI.

\section{FORMULATION OF THE PROBLEM}

The two-dimensional unsteady problem of an elastic plate impact on a thin layer of inviscid and incompressible liquid is considered. The plate motions and the liquid flow are described in the Cartesian coordinate system $(x, y)$ [see Fig. 1(a)]. The line $y=-H$ corresponds to the flat horizontal bottom of the liquid layer, and the line $y=0$ corresponds to the initial horizontal free surface of the liquid [see Fig. 1(a)]. Initially, $t=0$, the liquid is at rest, and the plate touches its free surface at a single point, which is taken as the origin of the Cartesian coordinate system. The plate is flat and corresponds to the interval $x=s \cos \alpha_{0}, y=s \sin \alpha_{0}$, where $0 \leq s \leq L, L$ is the plate length, $\alpha_{0}$ is the angle of the plate initial inclination to the horizontal surface of the liquid [see Fig. 1(a)], and $\alpha_{0}$ is positive and small in the present study. The distance $s$ of a plate element from the left edge of the plate is taken as the Lagrangian coordinate of this element. Initially, the plate elements have the velocity $-V_{0}$ in the vertical $y$ direction and the velocity $U_{0}$ in the horizontal $x$-direction, where both $V_{0}$ and $U_{0}$ are positive.

In this study, the layer thickness $H$ is much smaller than the plate length $L$, with $\varepsilon=H / L$ being a small parameter of the problem. The conditions of the impact are such that $\alpha_{0}=\varepsilon \tilde{\alpha}_{0}$ and $U_{0}=\lambda V_{0} / \varepsilon$, where $\tilde{\alpha}_{0}=O(1)$ and $\lambda=O(1)$ as $\varepsilon \rightarrow 0$. The penetration of the plate into the liquid layer is assumed comparable with the layer thickness, and the angle $\alpha(t)$ of the rigid rotation of the plate is of order of the initial angle $\alpha_{0}$ for any time. Coupled and strongly nonlinear motions of the liquid and the plate are of concern.

In order to justify the inviscid liquid model of impact on a thin liquid layer, we shall estimate the orders of the terms in the
Navier-Stokes equations and in the boundary conditions governing the flow. The velocity of the flow $\vec{u}(\vec{x}, t)$ is of the order of $O\left(V_{0} / \varepsilon\right)$. The viscous term $v \nabla^{2} \vec{u}$ in the Navier-Stokes equation, where $v$ is the kinematic viscosity of the liquid, can be neglected compared with the inertia term $\vec{u}_{t}$,

$$
\frac{\left|v \nabla^{2} \vec{u}\right|}{\left|\vec{u}_{t}\right|}=O\left(\frac{v T}{H^{2}}\right)
$$

when $v T / H^{2} \ll 1$. Here, $T=H / V_{0}$ is the characteristic time of the impact. For the conditions of the present calculations with the thickness of the water layer of $2 \mathrm{~cm}$ and vertical component of the impact velocity of order of $5 \mathrm{~m} / \mathrm{s}$, we have $T=4 \mathrm{~ms}, v=1.004 \times 10^{-6} \mathrm{~m}^{2} / \mathrm{s}$, and $v T / H^{2}=v / V_{0} H=10^{-5}$. This estimate justifies that the viscous effects can be neglected during impact events of short duration even for thin liquid layer.

In the present study, the horizontal speed of the plate is much higher than the critical speed $\sqrt{g H}$ of a signal propagation along the liquid layer. Then, the free boundary of the liquid is significantly deformed only near the advancing plate in the jet region [see Korobkin (1995)]. In this region, the free boundary of the liquid has a large curvature, which implies that the surface tension effects can be important there. The surface tension pressure in the jet region is estimated as $\sigma / H$, where $\sigma$ is the coefficient of surface tension of the liquid. For water at $20^{\circ}, \sigma=0.072 \mathrm{~N} / \mathrm{m}$. However, the dynamic pressure is also very high in the jet region. The dynamic pressure in the jet region is of the order of $\rho U_{0}^{2}$. Therefore, the ratio of the surface tension pressure to the dynamic pressure is estimated as

$$
\frac{\sigma / H}{\rho U_{0}^{2}}
$$

For the water layer of thickness $2 \mathrm{~cm}$ and the horizontal speed of the plate $U_{0}=25 \mathrm{~m} / \mathrm{s}$, we find that the surface tension pressure is about $5.8 \times 10^{-6}$ smaller than the dynamic pressure and it can be safely neglected.

In the wake behind the plate, the flow is governed, in general, by inertia of the liquid, its viscosity, and surface tension, with the gravity playing important role [see Alekseenko et al. (1994) and Chang and Demekhin (2002)]. The flow in the wake and a possible air entrainment in the wake depend on the plate motion and its elastic vibrations caused by the impact. However, the effect of the wake on the motion of the plate and the flow under the plate is minor because of high-speed motion of the plate along the layer.

In the present study, we are concerned with the liquid flow caused by the oblique impact of the elastic plate, mechanisms of the air entrainment under the plate, and the plate motions. The flow in the wake will be investigated in another follow-on paper.

\section{A. Structural part of the problem}

Let a plate element at a distance $s$ from the left edge of the plate have the coordinates $x=x_{p}(s, t)$ and $y=y_{p}(s, t)$ at time $t$. Here, $x_{p}(s, 0)=s \cos \alpha_{0}$ and $y_{p}(s, 0)=s \sin \alpha_{0}$. The motion of the element is described by the radius-vector, $\vec{r}=\vec{r}_{p}(s, t)=\left(x_{p}(s, t), y_{p}(s, t)\right)$, and is governed by the initial conditions,

$$
\vec{r}_{p}(s, 0)=s\left(\cos \alpha_{0}, \sin \alpha_{0}\right), \quad \frac{\partial \vec{r}_{p}}{\partial t}(s, 0)=\left(U_{0},-V_{0}\right),
$$


and external forces acting on this element. The external forces include [see Korobkin and Khabakhpasheva (2006)] (a) the hydrodynamic pressure in the wetted part of the plate, $p(x, y, t) \vec{n}$, which is calculated at the current position of the element, $x=x_{p}(s, t)$ and $y=y_{p}(s, t)$, and is acting in the normal direction to the element, where $\vec{n}(s, t)$ is the unit normal vector to the plate element directed from the fluid, (b) the force caused by bending stresses in the plate, $-E J\left(\partial^{4} w / \partial s^{4}\right) \vec{n}$, where $J=h_{p}^{3} / 12, h_{p}$ is the thickness of the plate, $E$ is the Young modulus of the plate material, $m=h_{p} \rho_{p}$ is the mass of the plate per unit length, $\rho_{p}$ is the density of the plate material, and $w(s, t)$ is the normal elastic deflection of the plate, and (c) the gravity force, $m \vec{g}$, where $\vec{g}=(0,-g)$, acting in the vertical direction downwards with $g$ being the gravity acceleration. Then, Newton's second law provides

$$
m \frac{\partial^{2} \vec{r}_{p}}{\partial t^{2}}=p\left(x_{p}(s, t), y_{p}(s, t), t\right) \vec{n}(s, t)-E J \frac{\partial^{4} w}{\partial s^{4}} \vec{n}(s, t)+m \vec{g},
$$

where $0 \leq s \leq L$ and $t>0$. Note that the elastic force in (1) is linearized in contrast to all other forces, which are in their original form. Equation (1) describes large rigid-body motions of the plate and its small elastic deflection. The elastic deflection $w(s, t)$ is considered here with respect to the rigid motions. Within this approximation, the normal $\vec{n}$ is calculated without accounting for the elastic deflection of the plate, $\vec{n}=(-\sin \alpha(t), \cos \alpha(t))$, where $\alpha(t)$ is the inclination of the rigid plate at time $t, \alpha(0)=\alpha_{0}$. Then,

$$
\begin{aligned}
& x_{p}(s, t)=x_{0}(t)+s \cos \alpha-w(s, t) \sin \alpha, \\
& y_{p}(s, t)=y_{0}(t)+s \sin \alpha+w(s, t) \cos \alpha,
\end{aligned}
$$

where $x_{0}(0)=0, y_{0}(0)=0, w(s, 0)=0$, and $w_{t}(s, 0)=0$. Projections of Eq. (1) onto the normal direction $\vec{n}$ to the plate and onto the tangential direction $\vec{\tau}=(\cos \alpha, \sin \alpha)$ yield

$$
\begin{gathered}
m \frac{\partial^{2} \vec{r}_{p}}{\partial t^{2}} \vec{n}+E J \frac{\partial^{4} w}{\partial s^{4}}=p\left(x_{p}(s, t), y_{p}(s, t), t\right)-m g \cos \alpha, \\
m \frac{\partial^{2} \vec{r}_{p}}{\partial t^{2}} \vec{\tau}=-m g \sin \alpha .
\end{gathered}
$$

Equations (4) and (5) provide the following equations for the functions $x_{p}(s, t)$ and $y_{p}(s, t)$ :

$$
\begin{aligned}
m \frac{\partial^{2} y_{p}}{\partial t^{2}}+E J \frac{\partial^{4} w}{\partial s^{4}} \cos \alpha & =p\left(x_{p}(s, t), y_{p}(s, t), t\right) \cos \alpha-m g, \\
\frac{\partial^{2} x_{p}}{\partial t^{2}} & =-\left(g+\frac{\partial^{2} y_{p}}{\partial t^{2}}\right) \tan \alpha .
\end{aligned}
$$

Note that $\partial^{4} w / \partial s^{4}=\left(\partial^{4} y_{p} / \partial s^{4}\right) / \cos \alpha$ [see Eq. (3)]. Therefore, (6) serves to determine the vertical coordinate $y_{p}(s, t)$ of the plate elements and (7) to determine the horizontal motion of the elements. Equation (6), written with respect to $y_{p}(s, t)$,

$$
\begin{gathered}
m \frac{\partial^{2} y_{p}}{\partial t^{2}}+E J \frac{\partial^{4} y_{p}}{\partial s^{4}}= \\
p\left(x_{p}(s, t), y_{p}(s, t), t\right) \cos \alpha-m g, \\
(0<s<L, t>0),
\end{gathered}
$$

should be solved subject to the edge conditions,

$$
\frac{\partial^{2} y_{p}}{\partial s^{2}}=0, \quad \frac{\partial^{3} y_{p}}{\partial s^{3}}=0 \quad(s=0, s=L),
$$

and the initial conditions,

$$
y_{p}(s, 0)=s \sin \alpha_{0}, \quad \frac{\partial y_{p}}{\partial t}(s, 0)=-V_{0} \quad(t=0) .
$$

The edge conditions (9) imply that the plate edges are free of bending stresses and shear forces correspondingly. Equation (7) is integrated in time subject to the initial conditions,

$$
x_{p}(s, 0)=s \cos \alpha_{0}, \quad \frac{\partial x_{p}}{\partial t}(s, 0)=U_{0} \quad(t=0) .
$$

Note that the functions $y_{p}(s, t)$ and $x_{p}(s, t)$ describe both rigid-body motions and elastic deflections together. Rigid-body rotation of the plate is described by the angle $\alpha(t)$, which appears in the right-hand sides of Eqs. (8) and (7). This angle can be determined at each step of the time integration by separating rigid and elastic motions.

We are concerned with such conditions of oblique impact of an elastic plate onto a thin liquid layer that both vertical rigid displacement of the plate and its elastic deflection are of the order of the layer depth $H$,

$$
\begin{gathered}
y_{p}=H \tilde{y}_{p}(\tilde{s}, \tilde{t}), \quad x=L \tilde{x}, \quad s=L \tilde{s}, \quad t=H \tilde{t} / V_{0}, \quad \varepsilon=H / L, \\
\alpha_{0}=\varepsilon \tilde{\alpha}_{0}, \quad \alpha=\varepsilon \tilde{\alpha}(\tilde{t}),
\end{gathered}
$$

where the dimensionless variables are denoted by tilde and $\varepsilon$ is a small parameter of the problem. Then, $\cos \alpha(t)=1+O\left(\varepsilon^{2}\right)$ in (8) and $\tan \alpha(t)=\varepsilon \tilde{\alpha}(\tilde{t})\left[1+O\left(\varepsilon^{2}\right)\right]$ in (7) in the leading order as $\varepsilon \rightarrow 0$. Therefore, one does not need to separate rigid and elastic motions to integrate equation (8). Indeed, in the leading order as $\varepsilon \rightarrow 0$, Eq. (7) provides $\partial^{2} x_{p} / \partial t^{2}=O\left(V_{0}^{2} / L\right)$, where the scales (12) were used and we assumed that $g H / V_{0}^{2}=O(1)$, which gives

$$
x_{p}(s, t)=s+U_{0} t+O\left(V_{0}^{2} t^{2} / L\right),
$$

with account for the initial conditions (11). The first two terms in (13) are of the same order $O(L)$ as $\varepsilon \rightarrow 0$ if the horizontal speed of the body $U_{0}$ is much greater than the vertical speed $V_{0}, U_{0}=\lambda V_{0} \varepsilon^{-1}$, where

$$
\lambda=\frac{U_{0}}{V_{0}} \frac{H}{L}=O(1)
$$

Then, $x_{p}=L \tilde{x}_{p}(\tilde{s}, \tilde{t})$ and

$$
\tilde{x}_{p}(\tilde{s}, \tilde{t})=\tilde{s}+\lambda \tilde{t}+O\left(\varepsilon^{2}\right),
$$

where $0 \leq \tilde{s} \leq 1$ and $\tilde{t}=O(1)$. Equation (8) describes the vertical motions of the elastic plate, $y_{p}(s, t)$, once the hydrodynamic pressure is known.

\section{B. Hydrodynamic part of the problem}

The position of the elastic plate in the global coordinate system is described by the equation

$$
y=H \tilde{y}_{b}(\tilde{x}, \tilde{t})
$$

in the dimensionless variables. In the leading order as $\varepsilon \rightarrow 0$, the flow is one-dimensional within the thin-layer approximation with the horizontal velocity of the flow beneath the plate, $V_{0} \varepsilon^{-1} \tilde{u}(\tilde{x}, \tilde{t})$, and the hydrodynamic pressure, $\rho V_{0}^{2} \varepsilon^{-2} \tilde{p}(\tilde{x}, \tilde{t})-\rho g H \tilde{y}_{b}(\tilde{x}, \tilde{t})$, satisfying 
the following equations of thin layer dynamics [see Khabakhpasheva and Korobkin (2013a; 2013b)]:

$$
\frac{\partial \tilde{y}_{b}}{\partial \tilde{t}}+\frac{\partial}{\partial \tilde{x}}\left(\tilde{u}(\tilde{x}, \tilde{t})\left[1+\tilde{y}_{b}(\tilde{x}, \tilde{t})\right]\right)=0, \quad \frac{\partial \tilde{u}}{\partial \tilde{t}}+\tilde{u} \frac{\partial \tilde{u}}{\partial \tilde{x}}=-\frac{\partial \tilde{p}}{\partial \tilde{x}}
$$

Note that the horizontal speed of the flow under the plate is of the same order as the horizontal speed of the plate.

Equation (16) should be solved in the region $\tilde{x}_{L}(\tilde{t})<\tilde{x}<\tilde{x}_{R}(\tilde{t})$, where $\tilde{x}=\tilde{x}_{L}(\tilde{t})$ corresponds to the left edge of the wetted part of the plate and $x=\tilde{x}_{R}(\tilde{t})$ is the coordinate of the leading edge of the wetted plate surface. We assume that the leading edge, $x_{R}(\tilde{t})$, propagates to the right at speed $\varepsilon^{-1} V_{0} x_{R}^{\prime}(\tilde{t})$ greater than the critical speed of the thin liquid layer, $\sqrt{g H}$. In this case, the liquid in front of the moving plate is not disturbed. The rest state, $\tilde{p}=0, \tilde{u}=0$, in $\tilde{x}>\tilde{x}_{R}(\tilde{t})$, is matched to the unsteady one-dimensional flow under the plate, which is described by Eq. (16) by a two-dimensional quasi-steady jet solution (Khabakhpasheva and Korobkin, 2013a). The matching leads to the following conditions for the speed of the leading edge:

$$
\frac{d \tilde{x}_{R}}{d \tilde{t}}=\frac{\tilde{u}\left(\tilde{x}_{R}, \tilde{t}\right)}{2\left(1-1 / \sqrt{1+\tilde{y}_{b}\left(\tilde{x}_{R}, \tilde{t}\right)}\right)},
$$

and the pressure there,

$$
\tilde{p}\left(\tilde{x}_{R}, \tilde{t}\right)=\frac{\tilde{u}^{2}\left(\tilde{x}_{R}, \tilde{t}\right)}{2\left(\sqrt{1+\tilde{y}_{b}\left(\tilde{x}_{R}, \tilde{t}\right)}-1\right)} .
$$

At the trailing edge, $\tilde{x}=\tilde{x}_{L}(\tilde{t})$, where the flow separates from the plate, the pressure is equal to the ambient pressure,

$$
\tilde{p}\left(\tilde{x}_{L}(\tilde{t}), \tilde{t}\right)=0 .
$$

The trailing edge, $\tilde{x}=\tilde{x}_{L}(\tilde{t})$, is initially at the left edge of the plate, $\tilde{x}_{L}(\tilde{t})=\lambda \tilde{t}$, with the pressure being positive (above the ambient pressure) in front of this edge, $\partial \tilde{p} / \partial \tilde{x}(\lambda \tilde{t}, \tilde{t})>0$. Note that the hydrodynamic pressure, $\tilde{p}(\tilde{x}, \tilde{t})$, can be below the ambient pressure but above the vapor pressure inside the contact region, $\tilde{x}_{L}(\tilde{t})<\tilde{x}<\tilde{x}_{R}(\tilde{t})$, but not near its edges. The scheme of the flow with the jet at the leading edge predicts positive pressure there [see condition (18)]. The inequality $\partial \tilde{p} / \partial \tilde{x}(\lambda \tilde{t}, \tilde{t})>0$ guarantees that the hydrodynamic pressure in front of the trailing edge is greater than the ambient pressure. It is possible that later $\partial \tilde{p} / \partial \tilde{x}(\lambda \tilde{t}, \tilde{t})$ approaches zero and becomes negative. Then, ventilation starts and the separation point, $\tilde{x}=\tilde{x}_{L}(\tilde{t})$, moves from the plate edge. In this case, the position of the separation point is determined using the Brillouin-Villat condition (Birkhoff and Zarantonello, 1957),

$$
\frac{\partial \tilde{p}}{\partial \tilde{x}}\left(\tilde{x}_{L}(\tilde{t}), \tilde{t}\right)=0 .
$$

This condition was used in the previous studies of oblique water impact to determine the position of the separation point on a smooth rigid surface (Vanden-Broeck, 1984; Tuck and Dixon, 1989; Faltinsen and Semenov, 2008; Khabakhpasheva and Korobkin, 2013a; and 2013b).

There is an important difference of the present problem of oblique plate impact from the problems of oblique impact of smooth surfaces studied in Khabakhpasheva and Korobkin (2013a; 2013b). For smooth surfaces, there is jetting at both edges of the contact region, at the leading and trailing edges, during an early stage. A low pressure zone appears first inside the contact region and expands with time reaching finally the trailing edge. At that instant, the liquid suddenly separates from the body surface with the trailing edge jumping to a new position defined by the condition (20). The motion of the trailing edge is not continuous when separation starts. In contrast to this scenario, the motion of the trailing edge is continuous for a body with a sharp edge as the plate of the present study. Therefore, we shall control the sign of the derivative $(\partial \tilde{p} / \partial \tilde{x})(\lambda \tilde{t}, \tilde{t})$ at the initial stage and allow the separation point to move to the right from the plate left edge when this derivative becomes negative.

The plate vertical position in the moving coordinates, $\tilde{y}_{p}(\tilde{s}, \tilde{t})$, and the plate position in the global coordinates, $\tilde{y}_{b}(\tilde{x}, \tilde{t})$, are related in the leading order as $\varepsilon \rightarrow 0$ by [see Eqs. (12), (14), and (15)]

$$
\tilde{y}_{b}(\tilde{x}, \tilde{t})=\tilde{y}_{p}(\tilde{x}-\lambda \tilde{t}, \tilde{t}) .
$$

Equations (16)-(21) and (8)-(10) describe a coupled problem of hydroelasticity of oblique impact of an elastic plate onto a thin liquid layer.

\section{Coupled problem of hydroelasticity}

There are no parameters in the dimensionless equations of the hydrodynamic part of the problem [(16)-(19)]. Only the structural part of the problem (8)-(10) depends on the parameters of the plate and conditions of the impact. In the leading order as $\varepsilon \rightarrow 0$, Eqs. (8)(10) in the dimensionless variables read

$$
\begin{aligned}
& M \frac{\partial^{2} \tilde{y}_{p}}{\partial \tilde{t}^{2}}+\frac{V_{\star}^{2}}{V_{0}^{2}} \frac{\partial^{4} \tilde{y}_{p}}{\partial \tilde{s}^{4}}= \tilde{p}\left(\tilde{s}+\lambda \tilde{t}, \tilde{y}_{p}(\tilde{s}, \tilde{t}), \tilde{t}\right)-\operatorname{Fr}^{-2}\left(\varepsilon^{2} \tilde{y}_{p}-M\right) \\
&(0<\tilde{s}<1, \tilde{t}>0), \\
& \frac{\partial^{2} \tilde{y}_{p}}{\partial \tilde{s}^{2}}=0, \quad \frac{\partial^{3} \tilde{y}_{p}}{\partial \tilde{s}^{3}}=0 \quad(\tilde{s}=0,1), \\
& \tilde{y}_{p}=\tilde{s} \tilde{\alpha}(0), \quad \frac{\partial \tilde{y}_{p}}{\partial \tilde{t}}=-1 \quad(\tilde{t}=0) .
\end{aligned}
$$

Here, $M=m \varepsilon^{2} /(\rho H)$ is the parameter of the plate inertia, Fr $=V_{0} / \sqrt{g H}$ is the Froude number, and $V_{*}^{2}=E J H \varepsilon^{2} /\left(\rho L^{4}\right)$ is a characteristic speed, which depends on elastic properties of the plate. For an aluminum plate with density $\rho_{p}=2700 \mathrm{~kg} / \mathrm{m}^{3}$, the Young modulus $E=68.3 \cdot 10^{9} \mathrm{~N} / \mathrm{m}^{2}$, length $L=10 \mathrm{~cm}$, and thickness $2 \mathrm{~mm}$ and a water layer of thickness $1 \mathrm{~cm}$, we find $V_{*}=0.2134 \mathrm{~m} / \mathrm{s}$. The interaction of such an elastic plate with the water layer is strong for the vertical speed of impact $V_{0}$ being of order $V_{\star}$. For the selected values of the impact parameters, we find $M=0.0054$ and $\mathrm{Fr}_{*}=V_{*} / \sqrt{g H} \approx 0.68$. We conclude that for the selected parameters, the hydrodynamic pressure is balanced by the elastic forces with the plate inertia and gravity being negligibly small. In the following, we keep all terms in $(22)$ and assume that $\mu=\left(V_{*} / V_{0}\right)^{2}=O(1)$.

It is convenient to integrate the hydrodynamic equations (16) with the boundary conditions (17)-(19) in the moving coordinate system using (21) and similar functions for the speed of the flow and the hydrodynamic pressure,

$$
\begin{gathered}
\tilde{u}(\tilde{x}, \tilde{t})=\lambda+U(\tilde{s}, \tilde{t}), \quad \tilde{p}(\tilde{x}, \tilde{t})=P(\tilde{s}, \tilde{t}), \quad \tilde{x}=\tilde{s}+\lambda \tilde{t}, \\
\tilde{x}_{R}(\tilde{t})=\lambda \tilde{t}+\tilde{c}_{R}(\tilde{t}), \quad \tilde{x}_{L}(\tilde{t})=\lambda \tilde{t}+\tilde{c}_{L}(\tilde{t}) .
\end{gathered}
$$


Here, $\tilde{c}_{L}(\tilde{t})=0$ during the initial stage when $(\partial \tilde{p} / \partial \tilde{x})(\lambda \tilde{t}, \tilde{t})$ $=\partial P / \partial \tilde{s}(0, \tilde{t})>0$. Tilde is dropped below.

Equation (16) keep their forms in the moving coordinates,

$$
\begin{gathered}
\frac{\partial Y}{\partial t}+\frac{\partial}{\partial s}(U(s, t) Y(s, t))=0, \\
\frac{\partial U}{\partial t}+U \frac{\partial U}{\partial s}=-\frac{\partial P}{\partial s}
\end{gathered}
$$

where $Y(s, t)=1+y_{p}(s, t)$. The parameter of the horizontal motion $\lambda$ does not appear in the equations of flow (26) and (27) and the equations of the plate deflection (22). It appears only in the matching conditions (17) and (18), which now read

$$
\begin{gathered}
\frac{d c_{R}}{d t}=\frac{\sqrt{Y_{R}(t)}\left(U_{R}(t)-\lambda\right)+2 \lambda}{2\left(\sqrt{Y_{R}(t)}-1\right)}, \\
P_{R}(t)=\frac{\left(\lambda+U_{R}(t)\right)^{2}}{2\left(\sqrt{Y_{R}(t)}-1\right)},
\end{gathered}
$$

where $P_{R}(t)=P\left(c_{R}(t), t\right), U_{R}(t)=U\left(c_{R}(t), t\right)$, and $Y_{R}(t)=1$ $+y_{p}\left(c_{R}(t), t\right)$. Initially $Y_{R}(0)=1$ and the speed of the leading contact point, $d c_{R} / d t$, is finite only if $U_{R}(0)=-\lambda$ [see (28)].

Integrating (26) in $s$ from $s$ to $c_{R}(t)$, we obtain

$$
U(s, t)=\frac{Y_{R}(t)}{Y(s, t)} U_{R}(t)+\frac{1}{Y(s, t)} \int_{s}^{c_{R}(t)} y_{p, t}\left(s_{0}, t\right) d s_{0} .
$$

The first derivatives of $U(s, t)$ are calculated using (26) and (30),

$$
\begin{gathered}
U_{s}(s, t)=-\frac{y_{p, t}(s, t)}{Y(s, t)}-U(s, t) \frac{y_{p, s}(s, t)}{Y(s, t)} \\
U_{t}(s, t)=\frac{Y_{R}(t)}{Y(s, t)} \frac{d U_{R}}{d t}+\frac{1}{Y(s, t)} \int_{s}^{c_{R}(t)} y_{p, t t}\left(s_{0}, t\right) d s_{0}+U^{(T)}(s, t), \\
U^{(T)}(s, t)=-\frac{y_{p, t}(s, t)}{Y(s, t)} U(s, t)+\frac{Y_{R}^{\prime}(t) U_{R}(t)+y_{p, t}\left(c_{R}, t\right) c_{R}^{\prime}(t)}{Y(s, t)}
\end{gathered}
$$

The derivative $d U_{R} / d t$ in (32) is related to the acceleration of the plate, $y_{p, t t}(s, t)$, by Eqs. (27) and (32) [see Sec. III]. Then, the derivative $U_{t}(s, t)$ is linearly dependent on the plate acceleration $y_{p, t t}(s, t)$ and nonlinearly dependent on both the shape of the plate, $y_{p}(s, t)$, and its speed, $y_{p, t}(s, t)$. Therefore, the pressure $P(s, t)$ given by $(27)$ is also linearly dependent on the plate acceleration. By using the hydrodynamic pressure $P(s, t)$ and the plate Eq. (22), we determine the plate acceleration, $y_{p, t t}(s, t)$, and then can integrate it in time subject to the initial conditions (24). The edge conditions (23) are included in the integration with the help of the normal mode method.

\section{NORMAL MODE METHOD}

In this method (Kvålsvold, 1994; Korobkin, 1998; Korobkin and Khabakhpasheva, 1998; and 2006), the shape function, $y_{p}(s, t)$, is sought in the form

$$
y_{p}(s, t)=\sum_{n=1}^{\infty} a_{n}(t) \psi_{n}(s),
$$

where $\psi_{n}(s)$ are the normal modes of the dry elastic plate and $a_{n}(t)$ are the coefficients to be determined. The normal modes are nonzero solutions of the eigen-value problem,

$$
\begin{gathered}
\frac{d^{4} \psi_{n}}{d s^{4}}=\lambda_{n}^{4} \psi_{n} \quad(0<s<1), \\
\frac{d^{2} \psi_{n}}{d s^{2}}=0, \quad \frac{d^{3} \psi_{n}}{d s^{3}}=0 \quad(s=0,1),
\end{gathered}
$$

where $\lambda_{n}$ is a spectral parameter, $n \geq 1$. There are two modes, $n=1$ and $n=2$, with $\lambda_{1}=\lambda_{2}=0$, which correspond to rigid motions of the plate,

$$
\psi_{1}(s)=1, \quad \psi_{2}(s)=2 \sqrt{3}\left(s-\frac{1}{2}\right) .
$$

The modes starting from $n=3$ correspond to elastic deflections of the plate,

$$
\begin{gathered}
\psi_{n}(s)=\cos \lambda_{n} s+\cosh \lambda_{n} s+B_{n}\left(\sin \lambda_{n} s+\sinh \lambda_{n} s\right), \\
B_{n}=-\frac{\cos \lambda_{n}-\cosh \lambda_{n}}{\sin \lambda_{n}-\sinh \lambda_{n}},
\end{gathered}
$$

where $\lambda_{n}$ are roots of the equation

$$
\cosh \lambda_{n} \cos \lambda_{n}=1 \quad(n \geq 3) .
$$

The modes (36) and (37) are orthonormal,

$$
\int_{0}^{1} \psi_{n}(s) \psi_{m}(s) d s=\delta_{n m},
$$

where $\delta_{n n}=1$ and $\delta_{n m}=0$ for $n \neq m$ and $n, m \geq 1$. The shape function (34) satisfies the edge conditions (23) because each term of the series (34) satisfies these conditions [see (35)].

Substituting (34) in the plate equation (22), multiplying both parts of the equation by $\psi_{k}(s), k \geq 1$, and integrating the result in $s$ from $s=0$ to $s=1$ using (39) provides

$$
\begin{aligned}
M \ddot{a}_{k}+\mu a_{k} \lambda_{k}^{4}= & \int_{c_{L}(t)}^{c_{R}(t)} P(s, t) \psi_{k}(s) d s \\
& -\varepsilon^{2} \operatorname{Fr}^{-2} \int_{c_{L}(t)}^{c_{R}(t)} y_{p}(s, t) \psi_{k}(s) d s+M \operatorname{Fr}^{-2} \delta_{k 1} .
\end{aligned}
$$

Note that the hydrodynamic pressure and the hydrostatic pressure, $-\mathrm{Fr}^{-2} \varepsilon^{2} y_{p}(s, t)$, in the right-hand side of $(22)$ are acting only in the wetted part of the plate, where $c_{L}(t)<s<c_{R}(t)$. Initial conditions for the system of ordinary differential equations (40) follow from (24),

$$
\begin{gathered}
a_{1}(0)=\frac{1}{2} \alpha_{0}, \quad a_{2}(0)=\frac{\alpha_{0}}{2 \sqrt{3}}, \quad a_{n}(0)=0 \quad(n \geq 3), \\
\dot{a}_{1}(0)=-1, \quad \dot{a}_{n}(0)=0 \quad(n \geq 2) .
\end{gathered}
$$

The contribution of the hydrostatic pressure to the plate deflection is proportional to the second integral in (40). This integral is 
evaluated by using (34),

$$
\begin{gathered}
\int_{c_{L}(t)}^{c_{R}(t)} y_{p}(s, t) \psi_{k}(s) d s=\sum_{n=1}^{\infty} a_{n}(t) R_{n k}(t), \quad R_{n k}(t)=\int_{c_{L}(t)}^{c_{R}(t)} \psi_{n}(s) \psi_{k}(s) d s, \\
R_{n k}(t)=\left(\psi_{k} \psi_{n}^{\prime \prime \prime}-\psi_{k}^{\prime} \psi_{n}^{\prime \prime}+\psi_{k}^{\prime \prime} \psi_{n}^{\prime}-\psi_{k}^{\prime \prime \prime} \psi_{n}\right)_{c_{L}}^{c_{R}} \quad(n \neq m) .
\end{gathered}
$$

The contribution of the hydrodynamic pressure to the plate deflection is described by the first integral in (40). This integral is calculated in the Appendix. In (A3), we set $x=s, \alpha(x)=\psi_{k}(s), p(x, t)$ $=P(s, t), y_{b}(x, t)=Y(s, t)$, and $\rho=1$. The following functions are introduced,

$$
\begin{gathered}
\bar{\psi}_{k}\left(c_{L}, s\right)=\int_{c_{L}}^{s} \psi_{k}\left(s_{0}\right) d s_{0}, \quad v_{k}(t)=\int_{c_{L}}^{c_{R}} \frac{\bar{\psi}_{k}\left(c_{L}, s\right) d s}{Y(s, t)}, \\
v_{0}(t)=\int_{c_{L}}^{c_{R}} \frac{d s}{Y(s, t)},
\end{gathered}
$$

where $v_{k}(t)$ stands for $D\left(c_{L}, c_{R}\right)$ [see (A11)] and $v_{0}(t)$ stands for $R\left(c_{L}\right.$, $\left.c_{R}\right)$ [see (A15)]. Next, we insert (34) in (A20), which gives

$$
\begin{aligned}
& \int_{c_{L}}^{c_{R}} P(s, t) \psi_{k}(s) d s= \sum_{m=1}^{\infty} \ddot{a}_{m}(t) B_{m k}(t)+\left(P_{L}+U_{L}^{2}\right) \frac{v_{k}}{v_{0}} \\
&+\left(P_{R}+U_{R}^{2}\right)\left(R_{1 k}-\frac{v_{k}}{v_{0}}\right) \\
&+\int_{c_{L}}^{c_{R}} U^{2}(s, t)\left\{\frac{Y_{s}(s, t)}{Y(s, t)}\left[\bar{\psi}_{k}\left(c_{L}, s\right)-\frac{v_{k}}{v_{0}}\right]\right. \\
&\left.-\psi_{k}(s)\right\} d s, \\
& B_{m k}=\frac{v_{m} v_{k}}{v_{0}}-\int_{c_{L}}^{c_{R}} \frac{\bar{\psi}_{m}\left(c_{L}, s\right) \bar{\psi}_{k}\left(c_{L}, s\right)}{Y(s, t)} d s .
\end{aligned}
$$

In (44), $P_{L}=0$ and $U_{L}(t)$ are given by (30) with $s=c_{L}(t)$. Equations (40) and (44) provide the second derivatives $\ddot{a}_{k}(t), k \geq 1$, as functions of the first derivatives $\dot{a}_{m}(t)$, the flow speed at the leading edge, $U_{R}(t)$, and the coordinates of the ends of the contact region, $c_{L}(t)$ and $c_{R}(t)$.

The flow velocity $U_{R}(t)$ at the leading edge, which appears in (28)-(30), satisfies the differential equation

$$
\begin{aligned}
\frac{d U_{R}}{d t}= & \frac{\left(P_{L}+U_{L}^{2}\right)-\left(P_{R}+U_{R}^{2}\right)}{v_{0} Y_{R}}-\frac{Y_{s}\left(c_{R}, t\right) c_{R}^{\prime} U_{R}+Y_{t}\left(c_{R}, t\right)\left[U_{R}+c_{R}^{\prime}\right]}{Y_{R}} \\
& -\frac{1}{v_{0} Y_{R}} \int_{c_{L}}^{c_{R}} U^{2}(s, t) \frac{Y_{s}(s, t)}{Y(s, t)} d s \\
& -\frac{1}{Y_{R}} \sum_{n=1}^{\infty} \ddot{a}_{n}(t)\left[\bar{\psi}_{n}\left(c_{L}, c_{R}\right)-\frac{v_{n}}{v_{0}}\right],
\end{aligned}
$$

which follows from (A8) and (A16). The system of differential equations (28), (40), (44), and (46) can be written in the following symbolic form:

$$
\begin{gathered}
\frac{d c_{R}}{d t}=G\left(c_{R}, U_{R}, \vec{a}\right), \\
\frac{d U_{R}}{d t}=K\left(c_{R}, c_{L}, U_{R}, \vec{a}, \vec{b}\right), \\
\frac{d \vec{a}}{d t}=\vec{b}, \\
\frac{d \vec{b}}{d t}=\vec{B}\left(c_{R}, c_{L}, U_{R}, \vec{a}, \vec{b}\right),
\end{gathered}
$$

where $\vec{a}=\left(a_{1}(t), a_{2}(t), \ldots\right)$ and $\vec{b}=\left(a_{1}^{\prime}(t), a_{2}^{\prime}(t), \ldots\right)$.

The system of ordinary differential equations (47) is truncated retaining a finite number of modes in (34) and integrated numerically in time subject to the initial conditions $(41)$ and $c_{R}(0)=0$ and $U_{R}(0)=-\lambda$. The system is integrated with $P_{L}(t)=0$ and $c_{L}(t)$ $=0$ during the early stage with separation of the liquid from the left edge of the plate if $\partial P / \partial s(0, t)>0$ at this edge. At each step of the integration, we compute $\partial P / \partial s(0, t)$ from (27) using (30)-(33) and $(46)$. Once $\partial P / \partial s(0, t)$ becomes negative, we solve the equation $\partial P / \partial s\left(c_{L}(t), t\right)=0$ with respect to $c_{L}$. Details of the numerical analysis are given in Sec. IV.

\section{NUMERICAL ALGORITHM}

In this section, several issues related to both the integration of the coupled equations of elastic plate impact in time and the presentation of the obtained results are covered.

\section{A. Initial asymptotic solution}

Initial asymptotic behavior of the flow is needed to start the numerical integration of the system (47). At $t=0$, both the denominators and numerators in (28) and (29) are equal to zero. In (46), $v_{0}(0)=0$, which does not allow us to calculate $d U_{R} / d t(0)$ and to start numerical integrations.

During the very early stage, $t \rightarrow 0$, we neglect elastic deformations of the plate and the hydrodynamic loads acting on the plate. Then, the plate equation in the dimensionless variables (12) and (15) reads

$$
y_{b}(x, t) \sim \alpha_{0}(x-\lambda t)-t
$$

where $\lambda t<x<A t, A=x_{R}^{\prime}(0)$. Note that tilde, which denotes dimensionless variables, has been dropped after Eq. (25). The penetration $y_{b}(x, t)=O(t)$ and $u(x, t)=O(t)$ as $t \rightarrow 0$. Equations of the flow (16) can be linearized. Then, at the leading order as $t \rightarrow 0$,

$$
\frac{\partial u}{\partial x} \sim-\frac{\partial y_{b}}{\partial t}=1+\alpha_{0} \lambda
$$

giving

$$
u(x, t) \sim\left(1+\alpha_{0} \lambda\right)(x-A t)+u_{R}(t)
$$

where

$$
u_{R}(t)=u\left(x_{R}(t), t\right) \sim B t \quad \text { as } \quad t \rightarrow 0 .
$$

The constants $A$ and $B$ are to be determined. The second equation in (16) after linearization and using (49) and (50) gives

$$
\frac{\partial p}{\partial x} \sim-\frac{\partial u}{\partial t}=A\left(1+\alpha_{0} \lambda\right)-B \quad(\lambda t<x<A t) .
$$


Equation (51) is integrated in $x$ subject to the end condition, $p(\lambda t, t)=0$,

$$
p(x, t)=\left[A\left(1+\alpha_{0} \lambda\right)-B\right](x-\lambda t) .
$$

The conditions at the leading edge (17) and (18) together with (48), (50), and (52) provide as $t \rightarrow 0$

$$
\begin{aligned}
A & =\lim _{t \rightarrow 0}\left\{\frac{u_{R}(t)}{2 y_{b}\left(x_{R}, t\right)} \sqrt{1+y_{b}\left(x_{R}, t\right)}\left[1+\sqrt{1+y_{b}\left(x_{R}, t\right)}\right]\right\} \\
& =\lim _{t \rightarrow 0}\left\{\frac{u_{R}(t)}{y_{b}\left(x_{R}, t\right)}\right\}
\end{aligned}
$$

and

$$
\begin{gathered}
A=\frac{B}{\alpha_{0}(A-\lambda)-1}, \\
p\left(x_{R}, t\right) \sim\left[A\left(1+\alpha_{0} \lambda\right)-B\right](A-\lambda) t \sim \frac{B^{2} t^{2}}{y_{b}\left(x_{R}, t\right)} \sim \frac{B^{2} t}{\alpha_{0}(A-\lambda)-1}
\end{gathered}
$$

and

$$
\left[A\left(1+\alpha_{0} \lambda\right)-B\right](A-\lambda)=\frac{B^{2}}{\alpha_{0}(A-\lambda)-1} .
$$

The solution of the system (53) and (54) is

$$
\begin{aligned}
& A=\lambda+\frac{3}{4 \alpha_{0}}+\frac{1}{4 \alpha_{0}} \sqrt{9+8 \alpha_{0} \lambda}, \\
& B=\frac{1}{4} \lambda+\frac{3}{8 \alpha_{0}}+\frac{1+2 \alpha_{0} \lambda}{8 \alpha_{0}} \sqrt{9+8 \alpha_{0} \lambda},
\end{aligned}
$$

where it was used that $y_{b}\left(x_{L}(t), t\right)>0$ at this early stage.

Therefore, the initial conditions for the system (47) are

$$
\begin{aligned}
& c_{R}(0)=0, \quad U_{R}(0)=-\lambda, \quad a_{1}(0)=\frac{1}{2} \alpha_{0}, \quad a_{2}(0)=\frac{\alpha_{0}}{2 \sqrt{3}}, \\
& a_{n}(0)=0 \quad(n \geq 3), \quad b_{1}(0)=-1, \quad b_{n}(0)=0 \quad(n \geq 2),
\end{aligned}
$$

and the right-hand sides of the system (47) are set at the very beginning of integration to

$$
\begin{gathered}
G(0,-\lambda, 0)=A-\lambda, \quad K(0,0,-\lambda, 0,0)=B, \\
B_{1}(0,0,-\lambda, 0,0)=\mathrm{Fr}^{-2}, \quad B_{n}(0,0,-\lambda, 0,0)=0 \quad(n \geq 2),
\end{gathered}
$$

where $A$ and $B$ are given by Eq. (55).

\section{B. Brillouin-Villat condition}

To determine the position of the trailing edge of the wetted part of the plate, $s=c_{L}(t)$, the derivative $\partial P / \partial s\left(c_{L}(t), t\right)$ is needed. Initially, $c_{L}(t)=0$ and $\partial P / \partial s(0, t)>0$ with separation of the liquid at the left edge of the plate. Setting $s=c_{L}(t)$ in (27) and using (30)-(33), we find

$$
\begin{aligned}
v_{0} Y_{L}(t) & \frac{\partial P}{\partial s}\left(c_{L}(t), t\right) \\
= & P_{R}+U_{R}^{2}+2 v_{0} U_{L} Y_{t}\left(c_{L}, t\right)+U_{L}^{2}\left(v_{0} Y_{s}\left(c_{L}, t\right)-1\right) \\
& +\int_{c_{L}}^{c_{R}} U^{2}(s, t) \frac{Y_{s}(s, t)}{Y(s, t)} d s-\sum_{n=1}^{\infty} \ddot{a}_{n}(t) v_{n} .
\end{aligned}
$$

The integral and the series in (58) have been calculated for the right-hand side of (46). Therefore, calculation of the pressure derivative at the trailing edge (58) at each step of time integration does not require significant extra computations.

If at a certain time instant $t_{*}$, the formula (58) for the first time gives $\partial P / \partial s\left(0, t_{*}\right)<0$, then we introduce $c_{L}>0$ and solve the equation $\partial P / \partial s\left(c_{L}, t_{*}\right)=0$ by Newton's iterative method. Actually, we solve equation $F\left(c_{L}, t_{*}\right)=0$, where $F\left(c_{L}, t_{*}\right)$ is the right-hand side of (58).

The derivative $\left(\partial F / \partial c_{L}\right)\left(c_{L}, t\right)$ required by Newton's method is calculated by differentiation of (58) in $c_{L}$ with setting $a_{n}, \dot{a}_{n}, \ddot{a}_{n}, c_{R}$, $U_{R}$, and $p_{R}$ to be independent of $c_{L}$,

$$
\begin{aligned}
\frac{\partial F}{\partial c_{L}}\left(c_{L}, t\right)= & \sum_{n=1}^{\infty} \ddot{a}_{n}(t) v_{n}+U_{L}^{2} v_{0}\left[Y_{s s}\left(c_{L}, t\right)-2 \frac{\left[Y_{s}\left(c_{L}, t\right)\right]^{2}}{Y\left(c_{L}, t\right)}\right] \\
& +U_{L} v_{0}\left[2 Y_{s t}\left(c_{L}, t\right)-4 \frac{Y_{t}\left(c_{L}, t\right) Y_{s}\left(c_{L}, t\right)}{Y\left(c_{L}, t\right)}\right] \\
& -2 v_{0} \frac{\left[Y_{t}\left(c_{L}, t\right)\right]^{2}}{Y\left(c_{L}, t\right)} .
\end{aligned}
$$

For $t>t_{*}$, the coordinate $c_{L}$ of the trailing edge is considered as a parameter of the system (47), which is determined by the equation $\partial P / \partial s\left(c_{L}, t\right)=0$, using Newton's iterative method at each time step. In the present cases, the left end of the wetted part of the plate, $s=c_{L}(t)$, always moves to the right along the plate toward the leading edge. It is possible that the trailing edge $s=c_{L}(t)$ changes the direction of its motion and moves toward the left edge of the plate. Such cases were detected in Reinhard et al. (2013) and treated by including a jet region at $s=c_{L}(t)$. A similar treatment is possible in the present problem with shallow water as well, where the matching conditions (17) and (18) are imposed in this case also at the trailing edge.

The pressure distribution in the wetted part of the elastic plate is calculated by the formula

$$
\begin{aligned}
P(s, t)= & {\left[Y\left(c_{L}, t\right) \frac{\partial P}{\partial s}\left(c_{L}, t\right)-2 U_{L}(t) Y_{t}\left(c_{L}, t\right)+U_{L}^{2}(t) Y_{s}\left(c_{L}, t\right)\right] } \\
& \times \int_{c_{L}}^{s} \frac{d s_{0}}{Y\left(s_{0}, t\right)}+U_{L}^{2}(t)-U^{2}(s, t)-\int_{c_{L}}^{s} U^{2}\left(s_{0}, t\right) \frac{Y_{s}\left(s_{0}, t\right)}{Y\left(s_{0}, t\right)} d s_{0} \\
& +\sum_{n=1}^{\infty} \ddot{a}_{n}(t) \int_{c_{L}}^{s} \frac{\bar{\psi}_{n}\left(c_{L}, s_{0}\right)}{Y\left(s_{0}, t\right)} d s_{0},
\end{aligned}
$$

where $\partial P / \partial s\left(c_{L}, t\right)=\partial P / \partial s(0, t)$ is given by (58) for $t<t_{*}$ and $\partial P / \partial s\left(c_{L}, t\right)=0$ for $t \geq t_{*}$. Note that the derivative $\partial P / \partial s\left(c_{L}, t\right)$ is calculated at each step of time integration of the system (47) but the pressure distribution $P(s, t)$, where $c_{L}(t)<s<c_{R}(t)$, is calculated only for some time steps selected in advance.

\section{Numerical integration}

The shape and position of the plate (34) are presented by the two terms, $n=1$ and $n=2$, of rigid motions and $N_{e}$ terms, $3 \leq n$ $\leq 2+N_{e}$, of elastic motions of the plate. $N_{e}=0$ corresponds to the problem of a rigid plate impact on a thin liquid layer. The effect of 
the number $N_{e}$ of the retained modes on the deflection of the plate and the strains in the plate is investigated by comparing solutions with different numbers of the modes.

Correspondingly, the system (47) is approximated by a finite truncated system involving $2\left(2+N_{e}\right)+2$ unknowns and equations. The finite system of ordinary differential equations is integrated by the explicit Euler method with time step $\triangle t=2 \times 10^{-4}$. All integrals in $s$, which appear in (30), (42)-(46), (58), and (60) are evaluated by the trapezoidal rule with the step $\triangle s=10^{-3} /\left(N_{e}+2\right)$, starting from $s=c_{L}$. The last panels of the integrals with length smaller than $\triangle s$ are treated separately. Equation $F\left(c_{L}, t\right)=0$ is solved by Newton's method with iterations

$$
c_{L}^{(n+1)}=c_{L}^{(n)}-\frac{F\left(c_{L}^{(n)}, t\right)}{\partial F / \partial c_{L}\left(c_{L}^{(n)}, t\right)}
$$

using (58) and (59), where $\mathrm{n}$ is the number of the iteration step and $c_{L}^{(0)}=c_{L}(t-\triangle t)$. The iterations are terminated when $\left|c_{L}^{(n+1)}-c_{L}^{(n)}\right|$ $<10^{-10}$. Note that the calculations are performed in the dimensionless variables.

\section{NUMERICAL RESULTS}

Calculations are performed for an aluminum plate with density $\rho_{p}=2670 \mathrm{~kg} / \mathrm{m}^{3}$ and the Young modulus $E_{a l}=68 \cdot 10^{9} \mathrm{~N} / \mathrm{m}^{2}$ and of length $L=10 \mathrm{~cm}$ and thickness $2 \mathrm{~mm}$ and the water layer of thickness $H=2 \mathrm{~cm}$. The water density is $1000 \mathrm{~kg} / \mathrm{m}^{3}$. For these conditions, we find $V_{*} \approx 0.6 \mathrm{~m} / \mathrm{s}, \varepsilon=0.2$, and $M=0.106$. The vertical initial speed of the plate is $V_{0}=5 \mathrm{~m} / \mathrm{s}$ in all calculations. Then, the pressure scale, $\rho V_{0}^{2} \varepsilon^{-2}$, is $625 \mathrm{kPa}$, the scale of the flow speed, $V_{0} \varepsilon^{-1}$, is $25 \mathrm{~m} / \mathrm{s}$ and the time scale, $H / V_{0}$, is $4 \mathrm{~ms}$.

To investigate the elastic effects and convergence of the solution in terms of the number of retained elastic modes $N_{e}$, calculations are performed with $N_{e}=3,5,7$ and for the rigid plate with $N_{e}=0$. The Young modulus $E$ of the plate material is artificially reduced by 100 and 1000 times in some calculations without changes of other parameters of the plate. The horizontal velocity of the plate is $U_{0}$ $=5 \mathrm{~m} / \mathrm{s}, 15 \mathrm{~m} / \mathrm{s}$, and $25 \mathrm{~m} / \mathrm{s}$, which gives $\lambda=0.2,0.6$, and 1 correspondingly. The initial angle of the plate inclination $\alpha_{0}$ is $3^{\circ}, 6^{\circ}$, and $10^{\circ}$. The pressure distributions and plate positions are plotted with the dimensionless time step 0.08 for inclination angles of $6^{\circ}$ and $10^{\circ}$ and with the time step 0.04 for the angle of $3^{\circ}$, where the duration of impact is relatively short.

The strain $\varepsilon(s, t)$ of the upper surface of the plate is calculated by the formula

$$
\varepsilon(s, t)=-\varepsilon_{s c} \frac{\partial^{2} y_{p}}{\partial s^{2}}(s, t),
$$

where $y_{p}(s, t)$ is given by series (34) with $N_{e}+2$ terms and the strain scale is $\varepsilon_{s c}=h_{p} H / L^{2}=0.002$. The strains are presented in microstrains $(\mu \mathrm{s})$, where $1 \mu$ s corresponds to the stress level of $68 \mathrm{kPa}$ for aluminum. Only the strain magnitude, $|\varepsilon(s, t)|$, is shown below.

The results of the calculations are presented in Figs. 2-10. The values of the parameters that vary in the calculations and their correspondence to the figures are given in Table I.
TABLE I. Parameters of calculations for Figs. 2-10.

\begin{tabular}{lccc}
\hline \hline$U_{0}(\mathrm{~m} / \mathrm{s})$ & $A_{0}(\mathrm{deg})$ & $E / E_{a l}$ & Figures \\
\hline 25 & 3 & 1 & 2 and 3 \\
25 & $3,6,10$ & 1 & 4 and 5 \\
$5,15,25$ & 10 & 1 & 6 and 7 \\
25 & 10 & $1,10^{-2}, 10^{-3}$ & 8 and 9 \\
$5,15,25$ & 10 & $10^{-3}$ & 10 \\
\hline \hline
\end{tabular}

The results presented in Figs. 2 and 3 are for the aluminum plate with $U_{0}=25 \mathrm{~m} / \mathrm{s}, \alpha_{0}=3^{\circ}$, and $N_{e}=7$. This is the reference case. The positions and shapes of the plate and pressure and strain distributions are shown in Fig. 2 in the dimensionless variables at six time
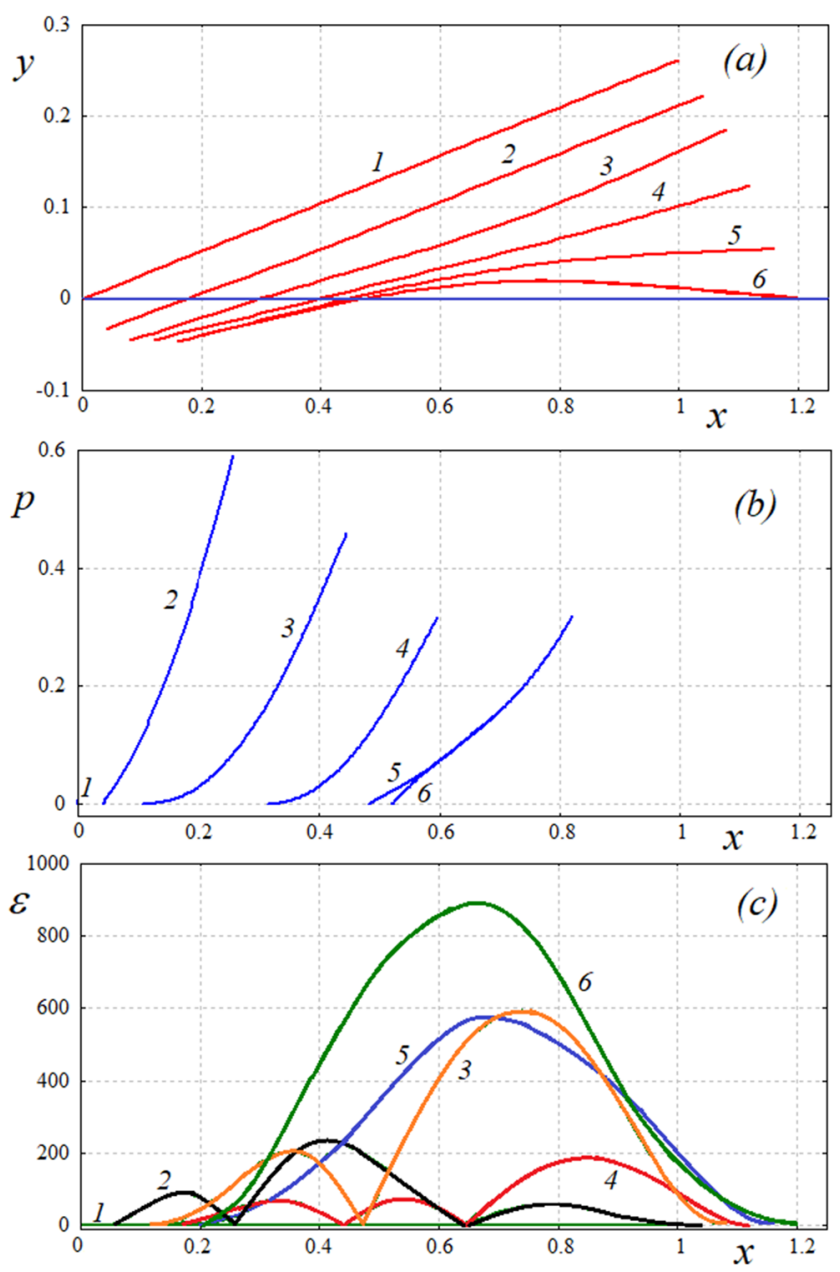

FIG. 2. Oblique impact by the aluminum plate: (a) positions of the plate in the dimensionless variables, (b) pressure distributions along the wetted part of the plate in the dimensionless variables, and (c) strains (in microstrains) along the plate. Lines 1-6 correspond to the dimensionless time instants $0,0.04,0.08,0.12$, 0.16 , and 0.2 . The time scale is $4 \mathrm{~ms}$. 

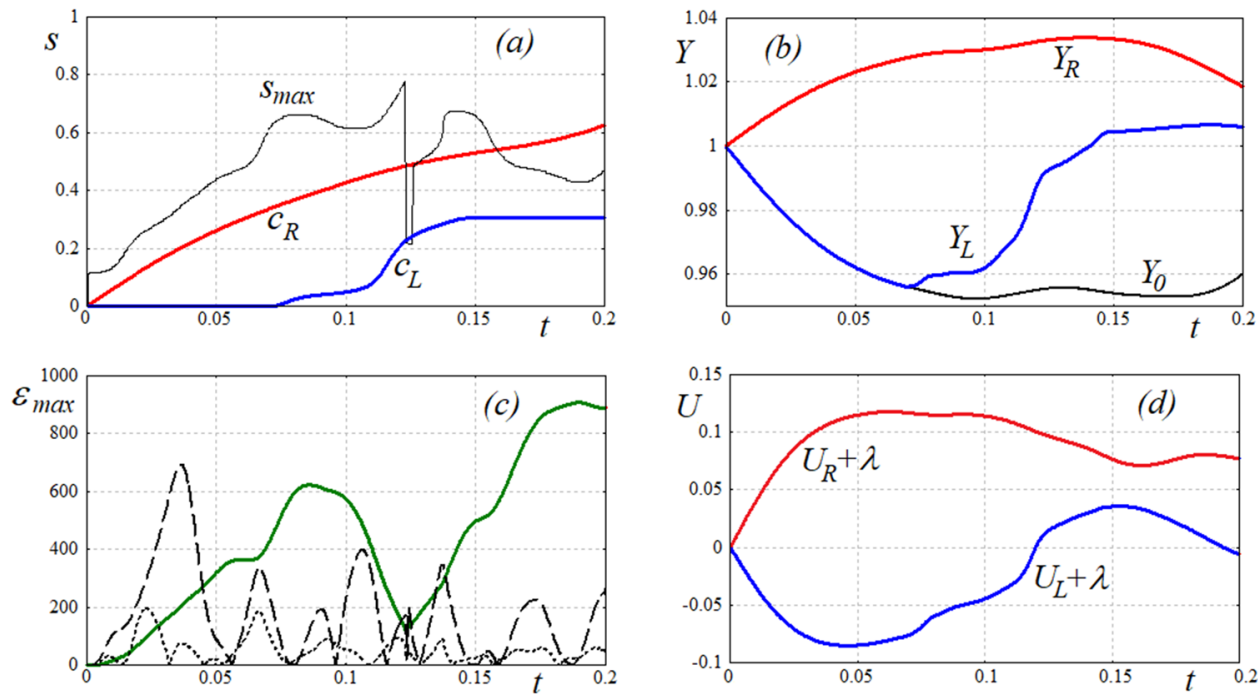

FIG. 3. Time evolutions of (a) the coordinates of the left, $c_{L}(t)$, and the right, $c_{R}(t)$, contact points of the wetted area and the point, $s_{\max }(t)$, where the maximum of strains is achieved. (b) Vertical elevations of the left contact point, $Y_{L}(t)$, the right contact point, $Y_{R}(t)$, and the left edge of the plate, $Y_{0}(t)=Y(0, t)$, above the liquid bottom. (c) Maximum strain, $\varepsilon_{\max }(t)$, (in microstrains) along the plate (solid line), the difference of $\varepsilon_{\max }(t)$ calculated with $N_{e}=7$ and with $N_{e}=3$ multiplied by 10 (dashed line), and the difference of $\varepsilon_{\max }(t)$ with $N_{e}=7$ and with $N_{e}=5$ multiplied by 10 (dotted line). (d) Dimensionless velocities of the flow at the left contact point, $U_{L}(t)+\lambda$, and at the right contact point, $U_{R}(t)+\lambda$. instants including the initial one. The calculations stop at $t=0.2$ when the leading edge of the plate touches the water surface trapping an air cavity under the plate [see Fig. 2(a)]. Elastic deflections of the plate are well visible. The pressure is always maximum at the leading edge of the wetted area, $s=c_{R}(t)$, where initially, it is linear in time [see (53) and Fig. 2(b)]. Note that the pressure along the wetted area [see Fig. 2(b)] and the strains [see Fig. 2(c)] are shown in the global dimensionless coordinate $x$. The tensile yield strength of the aluminum is $276 \mathrm{MPa}$, which corresponds to about 4000 microstrains. Figure 2(c) shows that the strains increase in time but stay well below the yield strain value.

More detailed information about this reference impact case is presented in Fig. 3. The positions of the left, $s=c_{L}(t)$, and right, $s=c_{R}(t)$, ends of the wetted part of the plate are shown in Fig. 3(a) by thick solid lines. One can see that the liquid separation from the plate occurs at the plate edge up to $t=0.07$. The length of the wetted part of the plate is always smaller than 0.4 , which is $4 \mathrm{~cm}$ in the dimensional variables. The length of the air cavity trapped under the plate at $t=0.2$ is $40 \%$ of the plate length.

The thicknesses of the liquid layer at three locations: the leading, $Y_{R}(t)$, and trailing, $Y_{L}(t)$, edges of the contact region and at the left edge of the plate, $Y_{0}(t)$, are shown in Fig. $3(\mathrm{~b})$ as functions of the dimensionless time $t$. It is seen that the penetration depth of the plate is rather small, less than $1 \mathrm{~mm}$, and therefore, the denominator $Y(s, t)$ can be approximated by 1 in (30)(33), (43)-(46), and (58)-(60). Then, the corresponding formulae and equations become simpler but keep their structure. The approximation of small penetration depth is not used in the present study.

The maximum strain, $\varepsilon_{\max }(t)=\max _{0<s<1}|\varepsilon(s, t)|$, is shown in Fig. 3(c) by the solid line. The strains are sensitive to the number of the retained elastic modes $N_{e}$. The difference between $\varepsilon_{\text {max }}(t)$ calculated with $N_{e}=7$ and $N_{e}=3$ and multiplied by 10 is shown in Fig. 3(c) by the dashed line, and a similar difference for $N_{e}=7$ and $N_{e}=5$ is shown by the dotted line. These curves demonstrate convergence of the strains with the number of modes $N_{e}$.

All calculations shown below are performed with $N_{e}=7$. The distance of the point, where the maximum strain is achieved, $s=s_{\max }(t)$, measured from the left edge of the plate is plotted in Fig. 3(a), $\varepsilon_{\text {max }}(t)=\left|\varepsilon\left(s_{\text {max }}(t), t\right)\right|$. The strains are maximum near the leading edge of the contact region, where the pressures are maximum [see Fig. 2(b)] and the plate curvature is highest [see Fig. 2(a)]. The dimensionless velocities of the global flow at the leading, $U_{R}(t)$ $+\lambda$, and the trailing, $U_{L}(t)+\lambda$, edges are shown in Fig. $3(\mathrm{~d})$. The scale of the flow velocity, $V_{0} \varepsilon^{-1}=25 \mathrm{~m} / \mathrm{s}$, is the same in all calculations. Note that the penetration depth is small in this reference case, which explains relatively small flow velocities compared with the horizontal speed of the plate. The flow under the leading edge of the contact region is accelerated to maximum speed of about $3 \mathrm{~m} / \mathrm{s}$ and then decays. At the trailing edge, the flow is accelerated in the direction opposite to the plate motion up to $2 \mathrm{~m} / \mathrm{s}$, which occurs before the trailing edge leaves the plate edge [see Fig. 3(a)]. Then, the flow velocity there decays and becomes positive in the direction of the plate motion at the final stage of the calculations. Starting from approximately $t=0.12$, the liquid under the plate moves in the direction of the plate motion but with a much smaller velocity.

Figures 4 and 5 compare impacts for different initial inclination angles: (a) reference case with $\alpha_{0}=3^{\circ}$, (b) $\alpha_{0}=6^{\circ}$, and (c) $\alpha_{0}$ $=10^{\circ}$. All others parameters are the same as in the reference case. It is seen that the impact duration is longer and the plate penetration is deeper for larger angles $\alpha_{0}$. The plate flexibility is less pronounced for $\alpha_{0}=6^{\circ}$ and $10^{\circ}$ with rigid-plate calculations, $N_{e}=0$, providing very close results except for the strains. The calculations are terminated for $\alpha_{0}=3^{\circ}$ and $6^{\circ}$ because the right edge of the plate enters the liquid trapping an air cavity. Note that the length of the cavity is $50 \%$ of the plate length for $\alpha_{0}=6^{\circ}$ and about $40 \%$ for $\alpha_{0}=3^{\circ}$. The contact region disappears at the end of calculations, $t=0.57$, for 

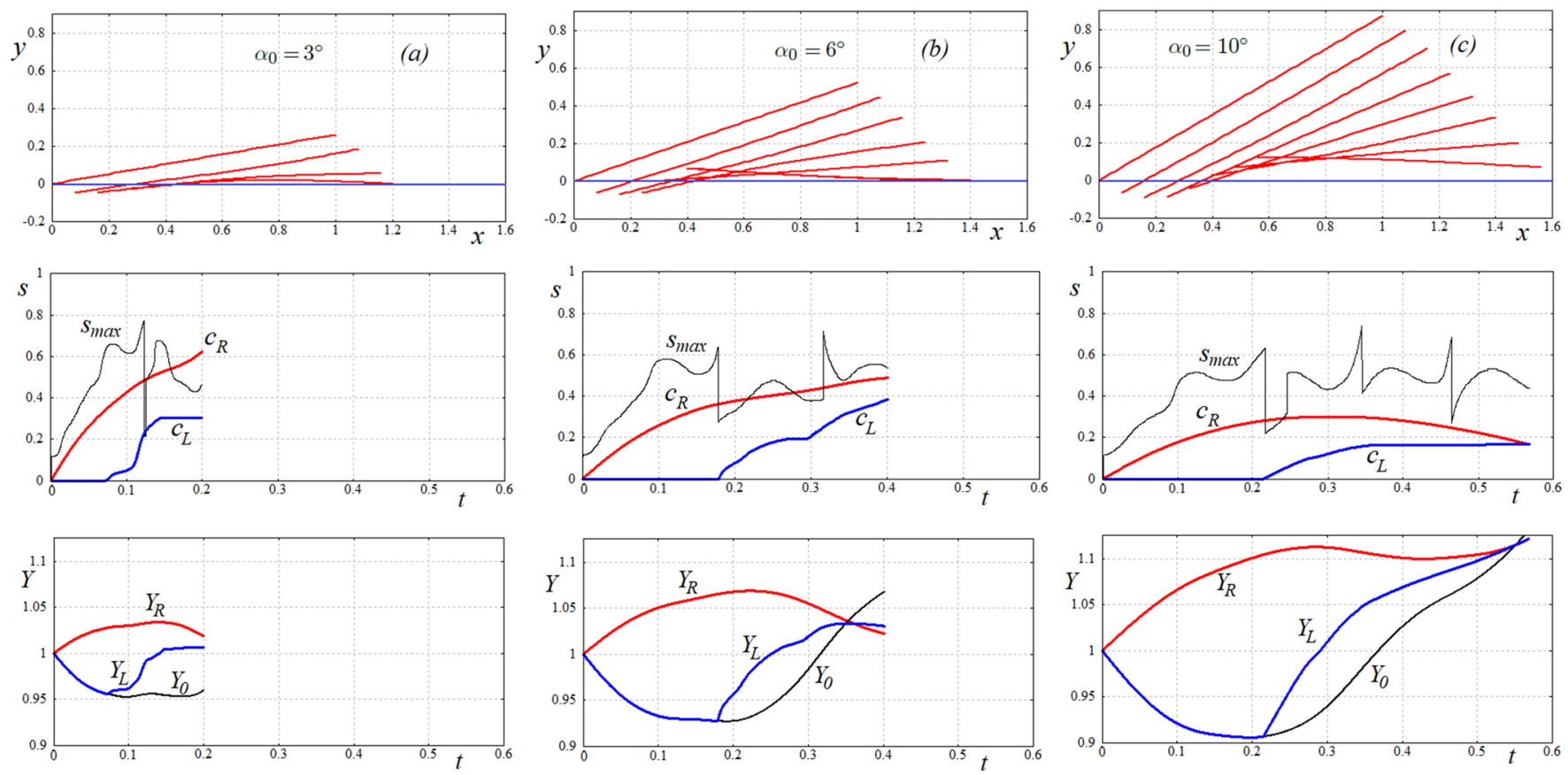

FIG. 4. Positions of the plate in the global dimensionless variables, the edges of the contact region, $c_{L}(t)$ and $c_{R}(t)$, together with the coordinate of the maximum strain, $s_{\max }(t)$, and the elevations of the moving points $c_{L}(t), c_{R}(t)$, and $s=0$ above the liquid bottom as functions of time for the initial inclination angle (a) $\alpha_{0}=3^{\circ}$, (b) $\alpha_{0}=6^{\circ}$, and (c) $\alpha_{0}=10^{\circ}$.
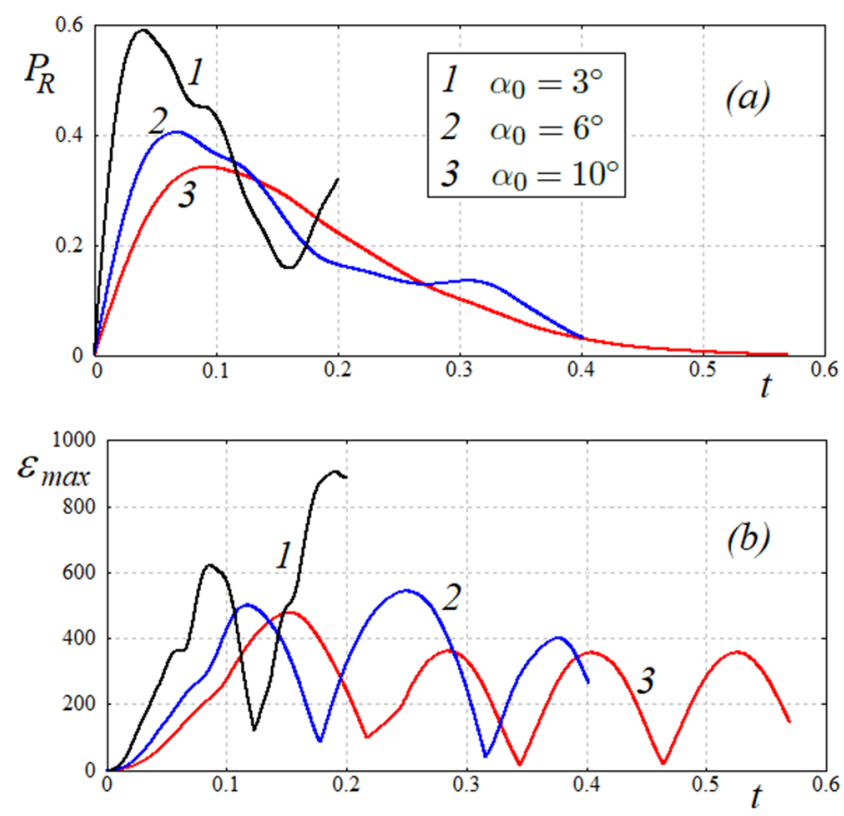

FIG. 5. (a) The pressure, $P_{R}(t)$, at the leading edge of the contact region and (b) the maximum strain, $\varepsilon_{\max }(t)$, as functions of the dimensionless time for the initial inclination angles of $\alpha_{0}=3^{\circ}$ (line 1), $\alpha_{0}=6^{\circ}$ (line 2), and $\alpha_{0}=10^{\circ}$ (line 3). $\alpha_{0}=10^{\circ}$, and then, the plate continues to move above the liquid layer until next impact. The inclination angle of the plate at the end of the impact is negative in this case. Therefore, it is expected that the plate will enter the liquid surface next time by its leading edge. This type of impact is not covered by the present model. The wetted part of the plate is short, less than $40 \%$ of the plate length, in all three cases. The trailing edge, $s=c_{L}(t)$, leaves the plate left edge during the impact. The left edge of the plate, $s=0$, is always the closest point of the plate to the liquid bottom. The free-surface elevation under the plate, where $0<s<c_{L}(t)$, is not studied here. Secondary impacts on the liquid are possible in this region.

Figure 5(a) presents the pressure, $P_{R}(t)$, at the leading edge of the contact region for the three initial angles of the plate inclination. This is the maximum pressure along the plate at each time instant [see Fig. 2(b)]. The pressure $P_{R}(t)$ initially quickly increases to its maximum value, which occurs well before the separation point, $s=c_{L}(t)$, starts to move from the left edge of the plate and then decays. The smaller the angle $\alpha_{0}$, the higher the maximum of $P_{R}(t)$.

The maximum strain, $\varepsilon_{\max }(t)$, is shown in Fig. 5(b) for the three initial angles of the plate inclination. It is seen that the first peak of $\varepsilon_{\text {max }}(t)$ is not necessary the highest one. The smaller the angle $\alpha_{0}$, the higher strains in the plate. The coordinates of the maximum strains, $s_{\max }(t)$, are shown in Fig. 4 together with the positions of the edges of the contact region. It is seen that the strains peak either in front of or near the leading edge, $s=c_{R}(t)$. The strains are much smaller than the yield strain value for aluminum. 

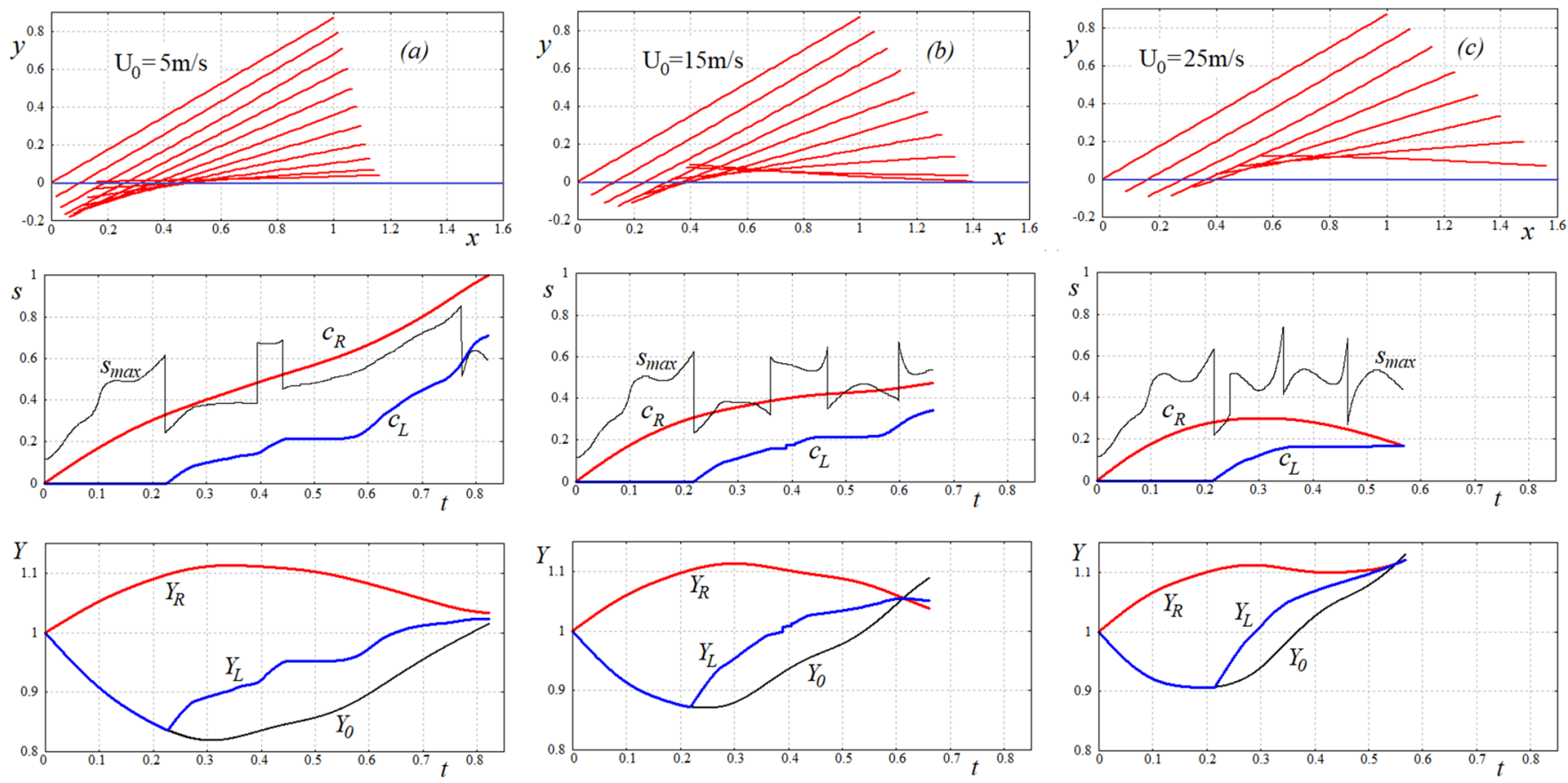

FIG. 6. Positions of the plate in the global dimensionless variables, the edges of the contact region, $c_{L}(t)$ and $c_{R}(t)$, together with the coordinate of the maximum strain $s_{\max }(t)$, and the elevations of the moving points $c_{L}(t), c_{R}(t)$, and $s=0$ above the liquid bottom as functions of time for the horizontal speed of the plate. (a) $U_{0}=5 \mathrm{~m} / \mathrm{s}$, (b) $U_{0}$ $=15 \mathrm{~m} / \mathrm{s}$, and $(\mathrm{c}) U_{0}=25 \mathrm{~m} / \mathrm{s}$.

Figures 4 and 5 demonstrate that the interaction between the plate and the liquid layer is longer for larger inclination angles $\alpha_{0}$. In the following, only impacts with $\alpha_{0}=10^{\circ}$ are considered.

Results of calculations for three different horizontal speeds of the plate, $U_{0}=5 \mathrm{~m} / \mathrm{s}, 15 \mathrm{~m} / \mathrm{s}, 25 \mathrm{~m} / \mathrm{s}$, and the initial inclination angle $\alpha_{0}=10^{\circ}$ are shown in Figs. 6 and 7. Other parameters of impacts are the same as in the reference case. Note that Figs. 4(c) and 6(c) are identical, and lines 3 in Fig. 5 are the same as lines 1 in Fig. 7. Figures 6 and 7 show that the larger the horizontal speed, the shorter the interaction time, 0.83 for $5 \mathrm{~m} / \mathrm{s}, 0.67$ for $15 \mathrm{~m} / \mathrm{s}$, and 0.57 for $25 \mathrm{~m} / \mathrm{s}$, and the smaller the penetration depth, which is up to $17 \%$ of the liquid depth for $5 \mathrm{~m} / \mathrm{s}, 13 \%$ for $15 \mathrm{~m} / \mathrm{s}$, and $10 \%$ for $25 \mathrm{~m} / \mathrm{s}$.

The calculations are terminated for $5 \mathrm{~m} / \mathrm{s}$, when the leading edge of the contact region arrives at the leading edge of the plate. The plate is almost parallel to the water surface at that time. Maximum length of the contact region does not exceed $40 \%$ of the plate length for this value of the horizontal speed. For $U_{0}=15 \mathrm{~m} / \mathrm{s}$, the calculations are terminated when the plate becomes inclined toward the liquid and the leading edge of the plate enters liquid trapping the air cavity. The cavity is long, about $50 \%$ of the plate length. For $U_{0}=25 \mathrm{~m} / \mathrm{s}$, the contact region shrinks at the end of calculation, and the plate losses its contact with the liquid and continues to move above the liquid layer until next impact. The separation point leaves the trailing edge of the plate at around $t=0.22$ for all values of the horizontal velocity $U_{0}$.
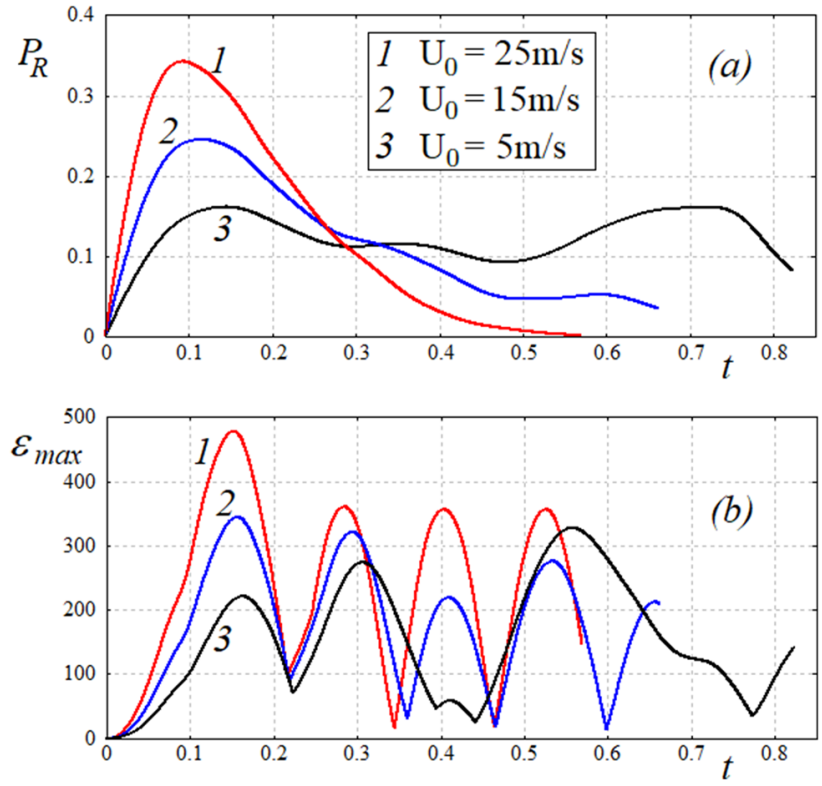

FIG. 7. (a) The pressure, $P_{R}(t)$, at the leading edge of the contact region and (b) the maximum strain, $\varepsilon_{\max }(t)$, as functions of the dimensionless time for the horizontal speed of the plate $U_{0}=25 \mathrm{~m} / \mathrm{s}$ (line 1), $U_{0}=15 \mathrm{~m} / \mathrm{s}$ (line 2), and $U_{0}=5 \mathrm{~m} / \mathrm{s}$ (line 3). 

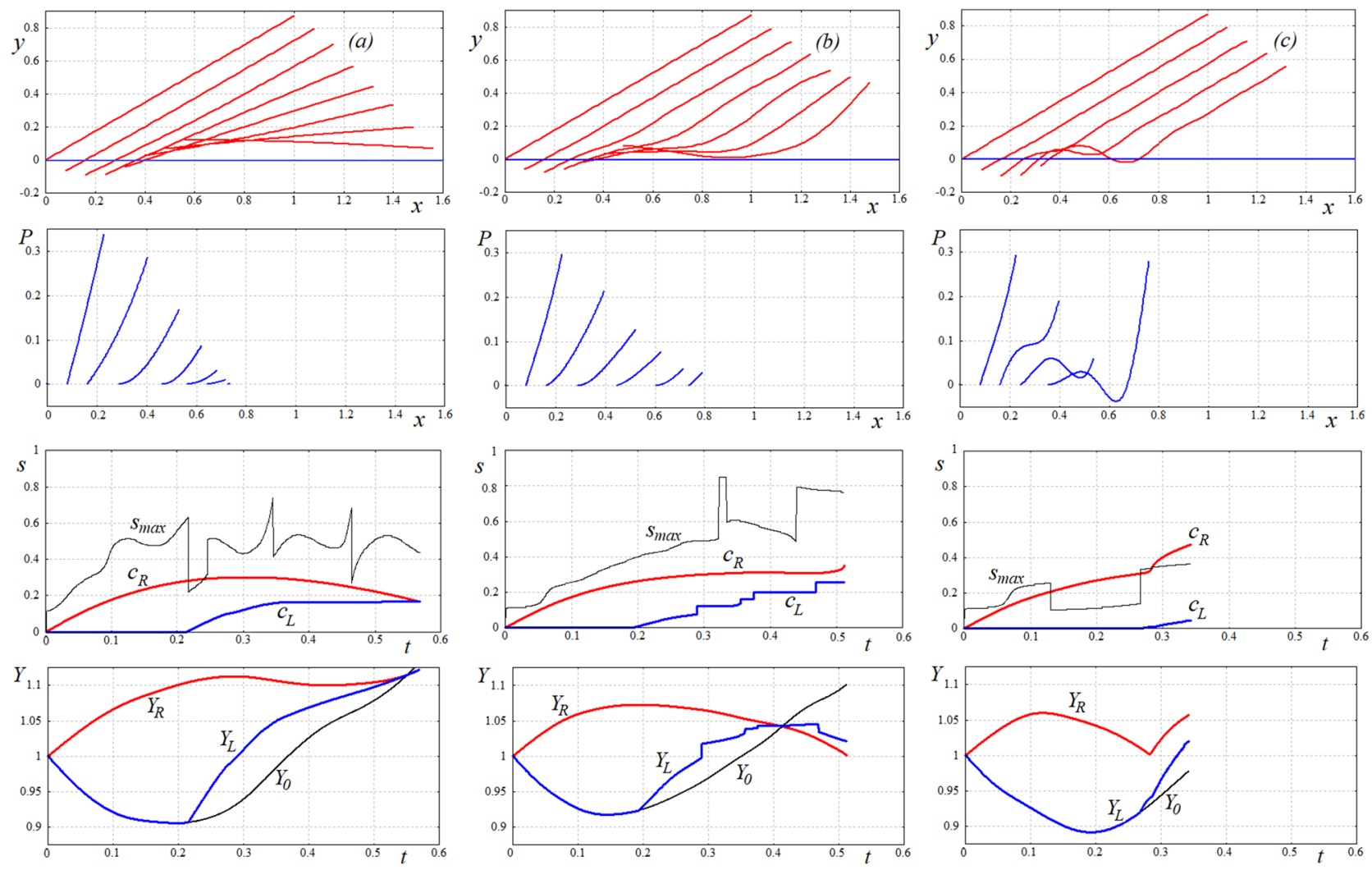

FIG. 8. Positions of the plate in the global dimensionless variables, pressure distributions along the contact region at the same time instants, the edges of the contact region, $c_{L}(t)$ and $c_{R}(t)$, together with the coordinate of the maximum strain, $s_{\max }(t)$, and the elevations of the moving points $c_{L}(t), c_{R}(t)$, and $s=0$ above the liquid bottom as functions of time for the Young modulus. (a) $E=E_{a l}$, (b) $E / E_{a l}=10^{-2}$, and (c) $E / E_{a l}=10^{-3}$

The dry part of the plate above the wake behind the plate is long for small $U_{0}$.

The coordinates of the maximum strain, $s_{\max }(t)$, are shown in Fig. 6 with respect to the coordinates of the edges of the contact region. The strains peak in the contact region for speed $5 \mathrm{~m} / \mathrm{s}$ near the leading edge $s=c_{R}(t)$ for $U_{0}=15 \mathrm{~m} / \mathrm{s}$ and in front of the leading edge for $U_{0}=25 \mathrm{~m} / \mathrm{s}$.

The pressure at the leading edge of the contact region, $P_{R}(t)$, and the maximum strain, $\varepsilon_{\max }(t)$, are depicted in Fig. 7. The pressure $P_{R}(t)$ is smaller for smaller speeds $U_{0}$ but lasts longer and decay slower. The strains are larger for larger $U_{0}$ but after their first peaks, their magnitude is weakly dependent on the horizontal speed of the plate.

Figures 8 and 9 demonstrate the effect of plate rigidity on the impact. Calculations are performed for the horizontal speed of the plate $U_{0}=25 \mathrm{~m} / \mathrm{s}$, initial inclination angle of the plate $\alpha_{0}=10^{\circ}$, and the plate rigidities $E_{a l}=68 \cdot 10^{9} \mathrm{~N} / \mathrm{m}^{2}, E=68 \cdot 10^{7} \mathrm{~N} / \mathrm{m}^{2}$, and $E=68 \cdot 10^{6} \mathrm{~N} / \mathrm{m}^{2}$. The longest time of interaction is observed for the most rigid plate [see Fig. 8(a)] where the plate bounces from the liquid at $t=0.57$. The plate also bounces from the liquid if the plate rigidity is reduced 100 times [see Fig. 8(b)], but the plate motion in this case is very different from the motion of the aluminum plate. The rear part of the plate moves upwards at the end of the
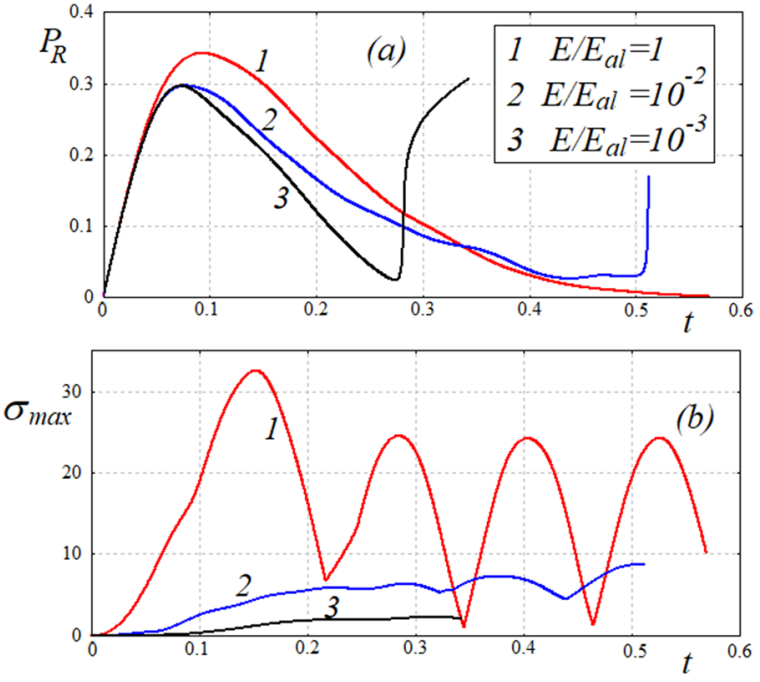

FIG. 9. (a) The pressure, $P_{R}(t)$, at the leading edge of the contact region. (b) The maximum stress in $\mathrm{GPa}\left(10^{9} \mathrm{~Pa}\right), \sigma_{\max }(t)$, as functions of the dimensionless time for the Young modulus $E=E_{a l}$ (line 1), $E / E_{a l}=10^{-2}$ (line 2), and $E / E_{a l}=10^{-3}$ (line 3). 
interaction stage, but the front part of the plate continues to move downwards. Figure 8(b) shows that another contact region, approximately at $x=0.9$, appears at the time or shortly after the original contact region disappears. The present model cannot describe two and more contact regions with air cavities trapped between them at the same time.

The motions of the softest plate [see Fig. 8(c)] are more complicated than for the rigid plate. The plate deflections occur mainly in the wetted part of the plate. The dry part of the plate keeps its initial motion. An important feature of this case is a negative pressure zone near the leading edge of the contact region. The pressure in this zone is $30 \mathrm{kPa}$ below the atmospheric pressure, which is $101.3 \mathrm{kPa}$. Therefore, the pressure in this zone is well above the vapor pressure and cavitation in this zone is not expected. The maximum pressure at the leading edge [see Fig. 9(a)] is approximately 0.3 in the dimensionless variables, which gives double atmospheric pressure in the dimensional variables. The dimensional (in GPa) bending stresses in the plate, $\sigma_{\max }(t)=E \varepsilon_{\max }(t)$, are reduced with the decrease in the plate rigidity [see Fig. 9(b)]. In all three cases [see Fig. 8], less than a half of the plate is wetted at the end of the calculations. The calculations are terminated for $E=E_{a l} / 1000$ [see Fig. 8(c)] when the plate touches the liquid surface in front of the leading edge of the contact region. The softest plate penetrates deeper into the liquid layer than more rigid plates.

The oblique impacts by the softest plate, $E=E_{a l} / 1000$, at $\alpha_{0}$ $=10^{\circ}$ with the horizontal speeds of $5 \mathrm{~m} / \mathrm{s}, 15 \mathrm{~m} / \mathrm{s}$, and $25 \mathrm{~m} / \mathrm{s}$ are depicted in Fig. 10. Note that Fig. 10(a) is the same as Fig. 8(c). The trailing edge of the plate contact region separates from the left edge of the plate quite late, which is because of the high flexibility of the plate. For $U_{0}=5 \mathrm{~m} / \mathrm{s}$, the calculations are terminated when the leading edge of the contact region arrives at the right edge of the plate [see Fig. 10(a)]. For $U_{0}=15 \mathrm{~m} / \mathrm{s}$ and $25 \mathrm{~m} / \mathrm{s}$, the calculations are terminated because the plate touches the liquid surface in front of the leading edge. The plate penetrates deeper for the lowest speed [see Fig. 10(a)] with low hydrodynamic pressure in the contact region. The trailing edge of the plate penetrates $25 \%$ of the layer thickness for $U_{0}=5 \mathrm{~m} / \mathrm{s}$. At this speed, the separation point, $s=c_{L}(t)$, moves from the left edge of the plate late at $t$ $=0.65$, and calculations are terminated at $t=0.8$, when the wetted region arrives at the right edge of the plate. The pressure in the contact region is low and is negative in some places. Deflections of the plate are well visible. The maximum strain is achieved inside the contact region for all speeds, except that of the very initial stage of the impact.
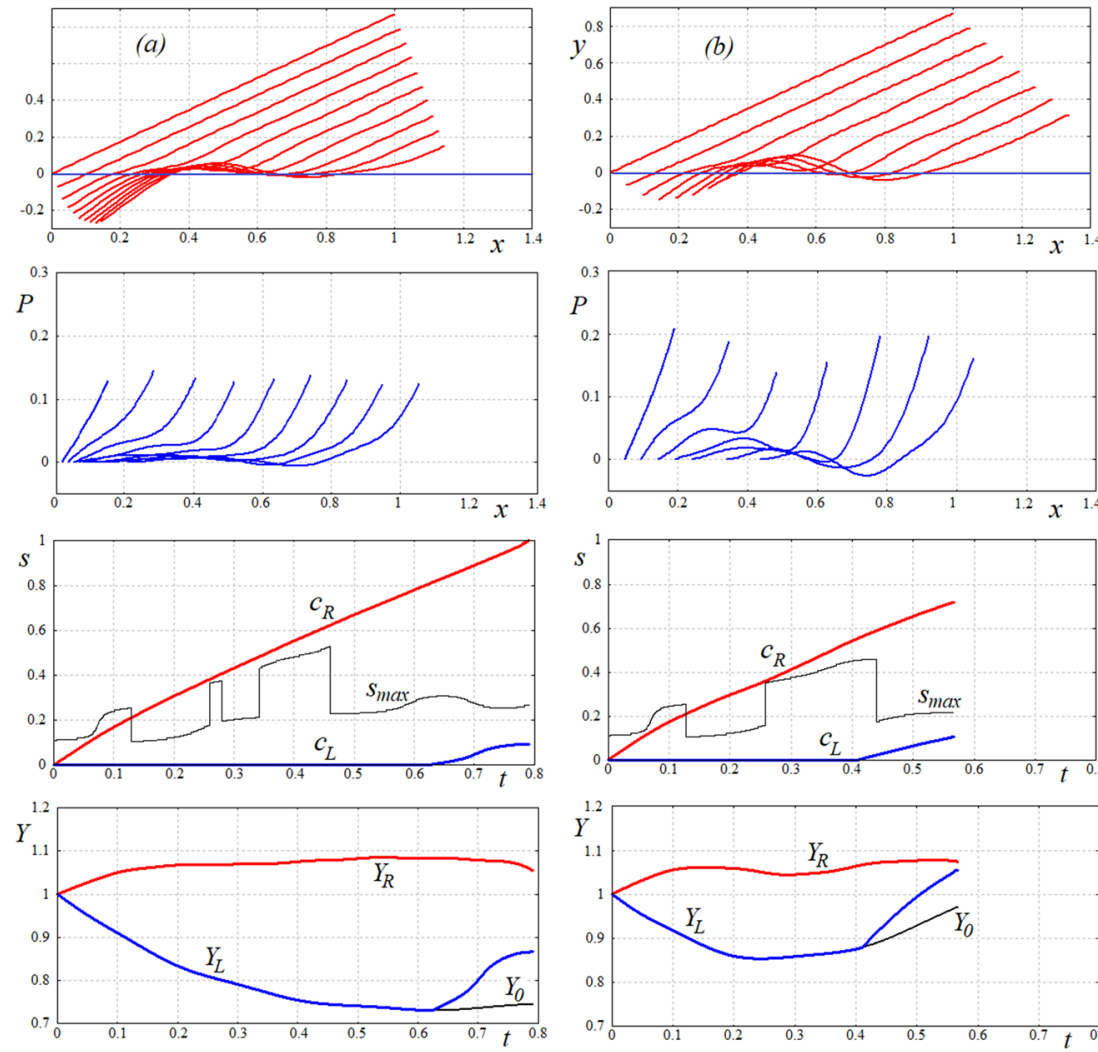
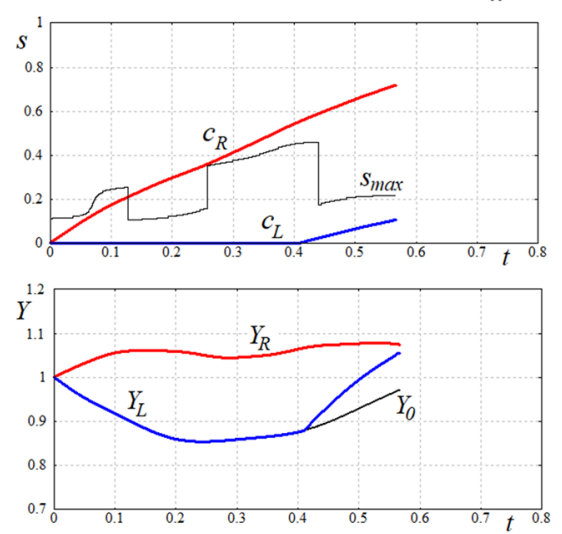
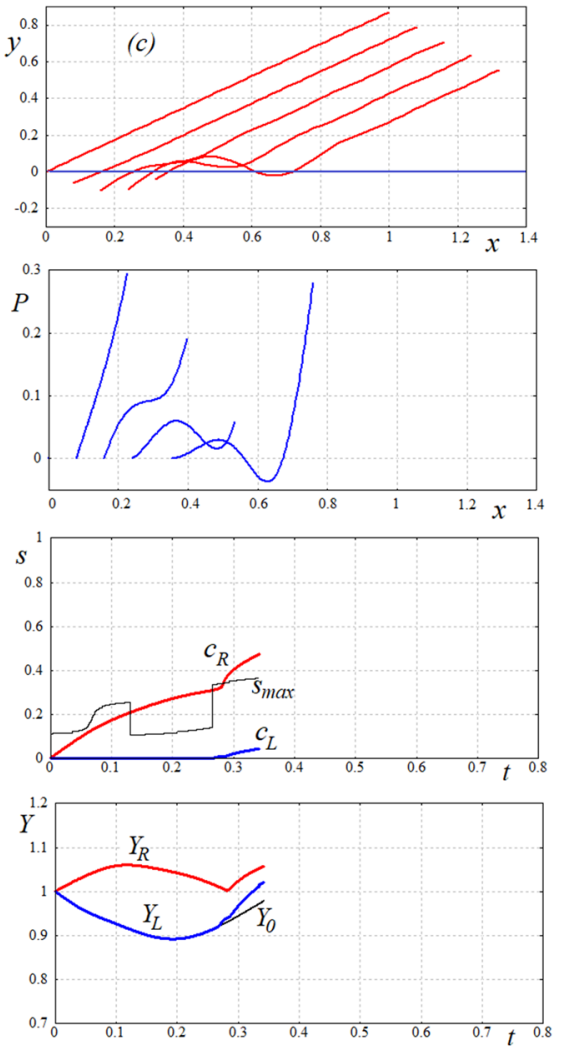

FIG. 10. Positions of the plate in the global dimensionless variables, pressure distributions along the contact region at the same time instants, the edges of the contact region, $c_{L}(t)$ and $c_{R}(t)$, together with the coordinate of the maximum strain, $s_{\max }(t)$, and the elevations of the moving points $c_{L}(t), c_{R}(t)$, and $s=0$ above the liquid bottom as functions of time for the plate with the Young modulus $E / E_{a l}=10^{-3}$ and the horizontal speed (a) $5 \mathrm{~m} / \mathrm{s}$, (b) $15 \mathrm{~m} / \mathrm{s}$, (c) $25 \mathrm{~m} / \mathrm{s}$. 


\section{CONCLUSION}

A model of oblique impact of an elastic plate on a thin liquid layer was presented. The problem was treated as coupled: the liquid flow beneath the plate, the plate elastic deflections, and rigid motions were determined at the sometime together with the position of the wetted part of the plate. At the leading edge of the wetted plate region, the one-dimension unsteady flow under the plate was matched to the two-dimensional and quasi-stationary jet flow. The position of the moving separation point (trailing edge of the contact region) was determined using the Brillouin-Villat condition of continuity of the pressure at this point together with its tangential derivative. The problem was reduced to a system of ordinary differential equations for the magnitudes of the rigid and elastic motions of the plate, the position of the leading edge of the contact region, and the flow speed at this point. The position of the trailing edge of the contact region was determined by iterations at each step of the time integration.

The obtained results demonstrate importance of elastic characteristics of the plate, as well as parameters of oblique impact such as velocity components and the inclination angle, on the plate motions and conditions of both cavitation and ventilation during the impact, which is in agreement with the experimental results by Iafrati and Grizzi (2019).

The results of the calculations showed that the interaction between the plate and the liquid layer is short for small angles of the initial plate inclination and/or large horizontal speeds of the plate. Elastic deflections of the plate are stronger pronounced for more flexible plates, impacting the liquid layer with low horizontal speeds and moderate inclination angles.

It was confirmed that air cavities can be trapped in front of the propagating contact region because of plate rotation and/or its elastic deflection. Negative pressures zones may occur in the contact region for flexible plates with low rigidities. The air is not trapped only if the plate bounces from the liquid surface before the leading edge of the plate enters the layer.

Another mechanism of the air entrainment by oblique elastic impacts can be due to the jet formed at the leading edge of the wetted region. Figure $3(\mathrm{~d})$ shows that the flow speed in the jet region is not monotonic and can oscillate, which may cause significant increase in the jet thickness with closing an air cavity in front of the moving plate. A similar but not identical increase in the thickness of the liquid layer may occur in the wake behind the plate because the volume flux at the trailing edge of the wetted region is not constant and oscillate [see Figs. 3(b) and 3(d)]. Note that the flow in the wake is affected by gravity, surface tension, and viscosity of the liquid [see Alekseenko et al. (1994) and Chang and Demekhin (2002)] in contrast to the flow under the plate where the inertia effects dominate. These other mechanisms of air entrainment in a thin liquid layer by an impacting deformable body will be investigated further and published in a separate paper.

\section{AUTHORS' CONTRIBUTIONS}

All authors contributed equally to this work.

\section{ACKNOWLEDGMENTS}

This work was supported by the Russian Science Foundation (Project No. 19-19-00287).

\section{APPENDIX: INTEGRALS OF THE PRESSURE IN THE PROBLEMS OF THIN-LAYER FLOWS}

A flow in a narrow gap between the rigid flat bottom, $y=0$, and a moving solid surface, $y=y_{b}(x, t)$, is described within the thin-layer approximation by the following equations:

$$
\begin{gathered}
\frac{\partial y_{b}}{\partial t}+\frac{\partial}{\partial x}\left[y_{b}(x, t) u(x, t)\right]=0, \\
\frac{\partial u}{\partial t}+u \frac{\partial u}{\partial x}=-\frac{1}{\rho} \frac{\partial p}{\partial x},
\end{gathered}
$$

where $\rho$ is the density of the liquid and $u(x, t)$ and $p(x, t)$ are the horizontal speed of the flow and the hydrodynamic pressure in the gap, $0<y<y_{b}(x, t), c_{L}(t)<x<c_{R}(t)$. In this Appendix, the functions $y_{b}(x$, $t), c_{L}(t)$, and $c_{R}(t)$ assumed are given. For convenience, the origin of the coordinate system in this section is taken at the bottom but not on the upper surface of the liquid at equilibrium as in the main body of the paper. This difference is minor and can be easily taken into account in using the results of this section in the main text. We also assume that $u(x, t)$ and $p(x, t)$ are given at the ends, $x=c_{L}$ and $x=c_{R}$, of the flow interval as $u_{L}(t), u_{R}(t), p_{L}(t)$, and $p_{R}(t)$, where $L$ stands for the left end and $R$ for the right end. We shall evaluate the integral

$$
L(t)=\frac{1}{\rho} \int_{c_{L}}^{c_{R}} p(x, t) \alpha(x) d x
$$

for any function $\alpha(x)$, which is not necessarily smooth.

To this aim, we introduce the function

$$
\bar{\alpha}\left(c_{L}, x\right)=\int_{c_{L}}^{x} \alpha\left(x_{0}\right) d x_{0}
$$

and integrate (A3) by parts using (A2),

$$
\begin{aligned}
L(t)= & \frac{1}{\rho} \int_{c_{L}}^{c_{R}} p(x, t) d\left[\bar{\alpha}\left(c_{L}, x\right)\right]=\frac{1}{\rho} p_{R}(t) \bar{\alpha}\left(c_{L}, c_{R}\right) \\
& +\int_{c_{L}}^{c_{R}}\left(u_{t}+u u_{x}\right) \bar{\alpha}\left(c_{L}, x\right) d x \\
= & \left(\frac{1}{\rho} p_{R}+\frac{1}{2} u_{R}^{2}\right) \bar{\alpha}\left(c_{L}, c_{R}\right)-\frac{1}{2} \int_{c_{L}}^{c_{R}} u^{2}(x, t) \alpha(x) d x \\
& +\int_{c_{L}}^{c_{R}} u_{t}(x, t) \bar{\alpha}\left(c_{L}, x\right) d x .
\end{aligned}
$$

To evaluate the integrals in (A4), we need the function $u(x, t)$ and its derivative $u_{t}(x, t)$. Integrating (A1) in $x$ from $x$ to $c_{R}$, we obtain

$$
y_{b}\left(c_{R}(t), t\right) u_{R}(t)-y_{b}(x, t) u(x, t)=-\int_{x}^{c_{R}} y_{b, t}\left(x_{0}, t\right) d x_{0} .
$$


Now we differentiate (A5) in time $t$,

$$
\begin{gathered}
{\left[y_{b, x}\left(c_{R}, t\right) c_{R}^{\prime}(t)+y_{b, t}\left(c_{R}, t\right)\right] u_{R}+y_{b}\left(c_{R}, t\right) \frac{d u_{R}}{d t}} \\
-y_{b, t}(x, t) u(x, t)-y_{b}(x, t) \frac{\partial u}{\partial t}(x, t) \\
=-\int_{x}^{c_{R}} y_{b, t t}\left(x_{0}, t\right) d x_{0}-y_{b, t}\left(c_{R}, t\right) c_{R}^{\prime}(t) .
\end{gathered}
$$

Prime stands for derivative in time. The derivative $y_{b, t}=-y_{b, x} u$ $-y_{b} u_{x}$ from (A1) is substituted in (A6), which gives

$$
B(t)+y_{b, x} u^{2}+y_{b} u u_{x}-y_{b} u_{t}=-\int_{x}^{c_{R}} y_{b, t t}\left(x_{0}, t\right) d x_{0},
$$

where

$$
\begin{aligned}
B(t)= & {\left[y_{b, x}\left(c_{R}, t\right) c_{R}^{\prime}+y_{b, t}\left(c_{R}, t\right)\right] u_{R}+y_{b}\left(c_{R}, t\right) u_{R}^{\prime}(t) } \\
& +y_{b, t}\left(c_{R}, t\right) c_{R}^{\prime}(t) .
\end{aligned}
$$

The derivative $u_{t}(x, t)$ is obtained from (A7),

$$
u_{t}(x, t)=u u_{x}+\frac{1}{y_{b}} \int_{x}^{c_{R}} y_{b, t t}\left(x_{0}, t\right) d x_{0}+\frac{y_{b x}}{y_{b}} u^{2}+\frac{B(t)}{y_{b}(x, t)} .
$$

Equation (A9) is used to evaluate the second integral in (A4),

$$
\begin{aligned}
\int_{c_{L}}^{c_{R}} u_{t}(x, t) \bar{\alpha}\left(c_{L}, x\right) d x \\
=\int_{c_{L}}^{c_{R}} \bar{\alpha}\left(c_{L}, x\right) d\left(\frac{u^{2}}{2}\right)+\int_{c_{L}}^{c_{R}} \frac{\bar{\alpha}\left(c_{L}, x\right)}{y_{b}(x, t)} \int_{x}^{c_{R}} y_{b, t t}\left(x_{0}, t\right) d x_{0} d x \\
\quad+\int_{c_{L}}^{c_{R}} \frac{y_{b, x}}{y_{b}} u^{2} \bar{\alpha}\left(c_{L}, x\right) d x+B(t) \int_{c_{L}}^{c_{R}} \frac{\bar{\alpha}\left(c_{L}, x\right) d x}{y_{b}(x, t)} \\
=\frac{1}{2} u_{R}^{2} \bar{\alpha}\left(c_{L}, c_{R}\right)-\frac{1}{2} \int_{c_{L}}^{c_{R}} u^{2} \alpha(x) d x \\
\quad+\int_{c_{L}}^{c_{R}} y_{b, t t}\left(x_{0}, t\right) \int_{c_{L}}^{x_{0}} \frac{\bar{\alpha}\left(c_{L}, x\right) d x}{y_{b}(x, t)} d x_{0} \\
\quad+\int_{c_{L}}^{c_{R}} \frac{y_{b, x}}{y_{b}} u^{2} \bar{\alpha}\left(c_{L}, x\right) d x+B(t) D\left(c_{L}, c_{R}\right)
\end{aligned}
$$

where

$$
D\left(c_{L}, x\right)=\int_{c_{L}}^{x} \frac{\bar{\alpha}\left(c_{L}, x_{0}\right) d x_{0}}{y_{b}\left(x_{0}, t\right)} .
$$

Substituting (A10) in (A4) and collecting similar terms, we find

$$
\begin{aligned}
L(t)= & \left(\frac{1}{\rho} p_{R}+u_{R}^{2}\right) \bar{\alpha}\left(c_{L}, c_{R}\right)+\int_{c_{L}}^{c_{R}} u^{2}(x, t)\left\{\frac{y_{b, x}}{y_{b}} \bar{\alpha}\left(c_{L}, x\right)-\alpha(x)\right\} d x \\
& +B(t) D\left(c_{L}, c_{R}\right)+\int_{c_{L}}^{c_{R}} y_{b, t t}\left(x_{0}, t\right) D\left(c_{L}, x_{0}\right) d x_{0} .
\end{aligned}
$$

The function $B(t)$, which is defined by (A8) and appears in (A9), can be obtained in another form by integrating (A2) from $c_{L}$ to $c_{R}$,

$$
\int_{c_{L}}^{c_{R}} u_{t}(x, t) d t+\frac{1}{2}\left(u_{R}^{2}-u_{L}^{2}\right)=-\frac{1}{\rho}\left(p_{R}-p_{L}\right)
$$

and using (A9) to evaluate the integral in (A13),

$$
\begin{aligned}
\int_{c_{L}}^{c_{R}} u_{t}(x, t) d x= & B(t) R\left(c_{L}, c_{R}\right)+\int_{c_{L}}^{c_{R}} u^{2} \frac{y_{b x}}{y_{b}} d x+\frac{1}{2}\left(u_{R}^{2}-u_{L}^{2}\right) \\
& +\int_{c_{L}}^{c_{R}} y_{b, t t}(x, t) R\left(c_{L}, x\right) d x
\end{aligned}
$$

where

$$
R\left(c_{L}, x\right)=\int_{c_{L}}^{x} \frac{d x_{0}}{y_{b}\left(x_{0}, t\right)}
$$

Equations (A13) and (A14) give the formula for $B(t)$,

$$
\begin{aligned}
B(t) R\left(c_{L}, c_{R}\right)= & -\int_{c_{L}}^{c_{R}} u^{2} \frac{y_{b, x}}{y_{b}} d x-\int_{c_{L}}^{c_{R}} y_{b, t t} R\left(c_{L}, x\right) d x+\left(\frac{1}{\rho} p_{L}+u_{L}^{2}\right) \\
& -\left(\frac{1}{\rho} p_{R}+u_{R}^{2}\right) .
\end{aligned}
$$

Finally, we substitute (A16) into (A12) and collect similar terms,

$$
\begin{aligned}
L(t)= & \left(\frac{1}{\rho} p_{R}+u_{R}^{2}\right) \bar{\alpha}\left(c_{L}, c_{R}\right)+\frac{D\left(c_{L}, c_{R}\right)}{R\left(c_{L}, c_{R}\right)}\left[\left(\frac{1}{\rho} p_{L}+u_{L}^{2}\right)-\left(\frac{1}{\rho} p_{R}+u_{R}^{2}\right)\right] \\
& +\int_{c_{L}}^{c_{R}} u^{2}(x, t)\left\{\frac{y_{b, x}}{y_{b}}\left[\bar{\alpha}\left(c_{L}, x\right)-\frac{D\left(c_{L}, c_{R}\right)}{R\left(c_{L}, c_{R}\right)}\right]-\alpha(x)\right\} d x \\
& +\int_{c_{L}}^{c_{R}} y_{b, t t}(x, t)\left\{D\left(c_{L}, x\right)-D\left(c_{L}, c_{R}\right) \frac{R\left(c_{L}, x\right)}{R\left(c_{L}, c_{R}\right)}\right\} d x .
\end{aligned}
$$

Formula (A17) can be presented in a symmetric form using a new function,

$$
S(x, t)=\frac{1}{y_{b}(x, t)}\left[\bar{\alpha}\left(c_{L}, x\right)-\frac{D\left(c_{L}, c_{R}\right)}{R\left(c_{L}, c_{R}\right)}\right] .
$$

The function $S(x, t)$ is such that

$$
\begin{aligned}
& \int_{c_{L}}^{x} S\left(x_{0}, t\right) d x_{0}=D\left(c_{L}, x\right)-\frac{D\left(c_{L}, c_{R}\right)}{R\left(c_{L}, c_{R}\right)} R\left(c_{L}, x\right), \\
& \int_{c_{L}}^{c_{R}} S\left(x_{0}, t\right) d x_{0}=0, \quad S\left(c_{L}, t\right)=-\frac{1}{y_{b}^{L}} \frac{D\left(c_{L}, c_{R}\right)}{R\left(c_{L}, c_{R}\right)} .
\end{aligned}
$$


Finally,

$$
\begin{aligned}
L(t)= & \left(\frac{1}{\rho} p_{R}+u_{R}^{2}\right) y_{b}^{R} S\left(c_{R}, t\right)-\left(\frac{1}{\rho} p_{L}+u_{L}^{2}\right) y_{b}^{L} S\left(c_{L}, t\right) \\
& +\int_{c_{L}}^{c_{R}} u^{2}(x, t)\left[y_{b x} S(x, t)-\alpha(x)\right] d x \\
& -\int_{c_{L}}^{c_{R}} S(x, t)\left(\int_{c_{L}}^{x} y_{b, t t}\left(x_{0}, t\right) d x_{0}\right) d x .
\end{aligned}
$$

\section{DATA AVAILABILITY}

The data that support the findings of this study are available within the article.

\section{REFERENCES}

Alekseenko, S. V., Nakoriakov, V. E., Pokusaev, B. G., and Fukano, T., Wave Flow of Liquid Films (Begell House, New York, 1994).

Batyaev, E. A. and Khabakhpasheva, T. I., "Initial stage of the inclined impact of a smooth body on a thin fluid layer," Fluid Dyn. 48(2), 211-222 (2013).

Batyaev, E. A. and Khabakhpasheva, T. I., "Oblique impact of an elongated threedimensional body on a thin liquid layer," J. Appl. Mech. Tech. Phys. 57(1), 163-172 (2016).

Belden, J., Hurd, R. C., Jandron, M. A., Bower, A. F., and Truscott, T. T., "Elastic spheres can walk on water," Nat. Commun. 7(1), 1-10 (2016).

Birkhoff, G. and Zarantonello, E., Jets, Wakes and Cavities (Academic Press, New York, 1957).

Chang, H. H. and Demekhin, E. A., Complex Wave Dynamics on Thin Films (Elsevier, 2002).

Cherdantsev, A. V., Hann, D. B., Hewakandamby, B. N., and Azzopardi, B. J., "Study of the impacts of droplets deposited from the gas core onto a gassheared liquid film," Int. J. Multiphase Flow 88, 69-86 (2017).

Faltinsen, O. M. and Semenov, Y. A., "The effect of gravity and cavitation on a hydrofoil near the free surface," J. Fluid Mech. 597, 371-394 (2008).

Hicks, P. D. and Purvis, R., "Air cushioning and bubble entrapment in threedimensional droplet impacts,” J. Fluid Mech. 649, 135-163 (2010).

Hicks, P. D. and Purvis, R., "Air cushioning in droplet impacts with liquid layers and other droplets," Phys. Fluids 23(6), 062104 (2011).

Hurd, R. C., Belden, J., Bower, A. F., Holekamp, S., Jandron, M. A., and Truscott, T. T., "Water walking as a new mode of free surface skipping," Sci. Rep. 9(1), 1-9 (2019).

Iafrati, A., "Experimental investigation of the water entry of a rectangular plate at high horizontal velocity," J. Fluid Mech. 799, 637-672 (2016).
Iafrati, A. and Grizzi, S., "Cavitation and ventilation modalities during ditching," Phys. Fluids 31(5), 052101 (2019).

Khabakhpasheva, T. I., "Fluid-structure interaction during the impact of a cylindrical shell on a thin layer of water," J. Fluids Struct. 25(3), 431-444 (2009).

Khabakhpasheva, T. I., "Impact of an elastic spherical shell on a thin fluid layer," Fluid Dyn. 50(2), 250-262 (2015).

Khabakhpasheva, T. I. and Korobkin, A. A., "Oblique impact of a smooth body on a thin layer of inviscid liquid,” Proc. R. Soc. A 469(2151), 20120615 (2013a).

Khabakhpasheva, T. I. and Korobkin, A. A., "Multiple oblique impacts on thin liquid layer with restoring forces," in Proceeding 28th International Workshop on Water Waves and Floating Bodies (L'Isle sur la Sourgue, France, 2013b), pp. 101-104, http://www.iwwwfb.org/Abstracts/iwwwfb28/iwwwfb28_26.pdf.

Korobkin, A., "Impact of two bodies one of which is covered by a thin layer of liquid,” J. Fluid Mech. 300, 43-58 (1995).

Korobkin, A., "Shallow-water impact problems," J. Eng. Math. 35(1-2), 233-250 (1999).

Korobkin, A. A., "Wave impact on the center of an Euler beam," J. Appl. Mech. Tech. Phys. 39(5), 770-781 (1998).

Korobkin, A. A. and Khabakhpasheva, T. I., "Plane problem of asymmetrical wave impact on an elastic plate,” J. Appl. Mech. Tech. Phys. 39(5), 782-791 (1998).

Korobkin, A. A. and Khabakhpasheva, T. I., "Regular wave impact onto an elastic plate,” J. Eng. Math. 55, 127-150 (2006).

Korobkin, A. A. and Khabakhpasheva, T. I., "Impact of elastic body on the deep and shallow water," in ASME 2013, 32nd International Conference on Ocean, Offshore and Arctic Engineering, 2013, Paper No. OMAE2013-11373, V009T12A052.

Kvålsvold, J., "Slamming loads on wetdecks of multihull vessels," in Proceedings of the International Conference on Hydroelasticity in Marine Technology, Trondheim, Norway (Balkema, Rotterdam, The Netherlands, 1994), Paper No. P1994-9, ISBN: 9054103876.

Liu, J., "Shallow-water skimming, skipping and rebound problems," Ph.D. thesis, UCL (University College London), UK, 2017.

Moradi, H., Rahbar Ranji, A., and Haddadpour, H., "Hydroelastic criterion for an inclined flat plate in vertical and oblique impacts," Appl. Ocean Res. 79, 173-183 (2018)

Reinhard, M., "Free elastic plate impact into water," Ph.D. thesis, UEA (University of East Anglia), UK, 2013.

Reinhard, M., Korobkin, A. A., and Cooker, M. J., "Water entry of a flat elastic plate at high horizontal speed," J. Fluid Mech. 724, 123-153 (2013).

Tkacheva, L. A., "Impact of a box with an elastic bottom on a thin liquid layer," J. Appl. Mech. Tech. Phys. 72(4), 427-436 (2008).

Tkacheva, L. A., "Impact of a body with a plane bottom on a thin liquid layer at a small angle," Fluid Dyn. 48(3), 352-365 (2013).

Tuck, E. O. and Dixon, A., “Surf-skimmer planing hydrodynamics,” J. Fluid Mech. 205, 581-592 (1989).

Vanden-Broeck, J.-M., "Numerical solutions for cavitating flow of a fluid with surface tension past a curved obstacle," Phys. Fluids 27(11), 2601-2603 (1984). 